\title{
ANALYTIC EXTENSION TECHNIQUES FOR UNITARY REPRESENTATIONS OF BANACH-LIE GROUPS
}

\author{
STÉPHANE MERIGON AND KARL-HERMANN NEEB
}

\begin{abstract}
Let $(G, \theta)$ be a Banach-Lie group with involutive automorphism $\theta, \mathfrak{g}=\mathfrak{h} \oplus \mathfrak{q}$ be the $\theta$-eigenspaces in the Lie algebra $\mathfrak{g}$ of $G$, and $H=\left(G^{\theta}\right)_{0}$ be the identity component of its group of fixed points. An Olshanski semigroup is a semigroup $S \subseteq G$ of the form $S=H \exp (W)$, where $W$ is an open $\operatorname{Ad}(H)$ invariant convex cone in $\mathfrak{q}$ and the polar map $H \times W \rightarrow S,(h, x) \mapsto h \exp x$ is a diffeomorphism. Any such semigroup carries an involution $*$ satisfying $(h \exp x)^{*}=(\exp x) h^{-1}$. Our central result, generalizing the Lüscher-Mack Theorem for finite dimensional groups, asserts that any locally bounded $*$ representation $\pi: S \rightarrow B(\mathcal{H})$ with a dense set of smooth vectors defines by "analytic continuation" a unitary representation of the simply connected Lie group $G_{c}$ with Lie algebra $\mathfrak{g}_{c}=\mathfrak{h}+i \mathfrak{q}$. We also characterize those unitary representations of $G_{c}$ obtained by this construction. With similar methods, we further show that semibounded unitary representations extend to holomorphic representations of complex Olshanski semigroups.
\end{abstract}

\section{INTRODUCTION}

There are many important results in the unitary representation theory of Lie groups related to analytic continuation. Here a key ingredient is the special case where $\pi: \mathbb{R} \rightarrow \mathrm{U}(\mathcal{H})$ is a strongly continuous unitary one-parameter group and $A$ its self-adjoint infinitesimal generator, i.e., $\pi(t)=e^{i t A}$ in the sense of measurable functional calculus. Then the unitary one-parameter group $\pi$ extends to a holomorphic one-parameter semigroup $\widehat{\pi}: \mathbb{C}_{+}=\mathbb{R}+i \mathbb{R}_{\geq 0} \rightarrow B(\mathcal{H})$ if and only if $A$ is bounded below. This extension then restricts to a locally bounded non-degenerate hermitian one-parameter semigroup $t \mapsto \widehat{\pi}(i t)$. Conversely, any such hermitian one-parameter group has a self-adjoint infinitesimal generator $-A$ and $e^{i z A}$ then yields an extension to $\mathbb{C}_{+}$, where the boundary values form a unitary one-parameter group. The key point of this picture is that self-adjoint operators $A$ whose spectrum is bounded below can be viewed as the infinitesimal generators of two objects: a unitary oneparameter group (Stone's Theorem) and a hermitian one-parameter semigroup of bounded operators (Hille-Yosida Theorem). This is the one-parameter context of what we are dealing with in the present paper for Banach-Lie groups.

We call a pair $(G, \theta)$ consisting of a Banach-Lie group $G$ and an involutive automorphism $\theta$ of $G$ a symmetric Banach-Lie group. We decompose its Lie algebra $\mathfrak{g}=\mathfrak{h} \oplus \mathfrak{q}$ into \pm 1 -eigenspaces of $\theta$ and write $H:=G_{0}^{\theta}$ for the identity component of the group of $\theta$-fixed points in $G$. Let $W \subseteq \mathfrak{q}$ be an open convex $\operatorname{Ad}(H)$-invariant cone for which the polar map

$$
H \times W \rightarrow H \exp (W), \quad(h, x) \mapsto h \exp x
$$

is a diffeomorphism onto an open subsemigroup $S:=S_{H}(W):=H \exp (W)$ of $G$. Then $S$ is an involutive semigroup with respect to the involution $s^{*}:=\theta(s)^{-1}$ which is called an Olshanski semigroup. In Appendix A we explain how to obtain such semigroups $S_{H^{\prime}}(W)$, where $H^{\prime}$ is a connected Lie group locally isomorphic to $H$ for which $H^{\prime}$ and $S_{H^{\prime}}(W)$ need not be contained in a Lie group (cf. [Ne92, Ex. II.13]). 
Our first main result (Theorem 3.7, proved in Section 4) is the following Banach version of the Lüscher-Mack Theorem ([LM75]): Let $\rho: S=S_{H}(W) \rightarrow B(\mathcal{H})$ be a non-degenerate strongly continuous $*$-representation of $S$ which is smooth in the sense that the space $\mathcal{H}^{\infty}$ of smooth vectors is dense. If $G_{c}$ is the simply connected Lie group with Lie algebra $\mathfrak{g}_{c}:=\mathfrak{h}+i \mathfrak{q}$, then there exists a smooth unitary representation $(\pi, \mathcal{H})$ of $G_{c}$ on $\mathcal{H}$ which is uniquely determined by the requirement that the unitary one-parameter groups corresponding to elements $x \in \mathfrak{h}$ are those obtained by "extending" $\rho$ to $H$ and the generators of the one-parameter groups corresponding to elements of $i W \subseteq i \mathfrak{q}$ are of the form $i \mathrm{~d} \rho(x)$, where $\mathrm{d} \rho(x)$ is the infinitesimal generator of the hermitian one-parameter semigroup $t \mapsto \rho(\exp t x)$. For finite dimensional groups this result is due to M. Lüscher and G. Mack ([LM75]; see also [HN93]). As their methods make heavy use of coordinates obtained from products of one-parameter groups, we have to develop a completely new approach in the Banach context. Actually our approach is more direct and uses only quite general methods, such as the criteria for the integrability of infinitesimal unitary representations of Banach-Lie algebras from [Mer10]. The one-parameter case described above corresponds to $G=\mathbb{R}, \theta(g)=-g, S=\mathbb{R}_{>0}$ and $G_{c}=i \mathbb{R}$.

An interesting, much less involved, special case arises if $S=G$ is the whole group. Then our assumption is that $G$ has a diffeomorphic polar decomposition $H \exp \mathfrak{q}$ and our theorem establishes a correspondence between $*$-representations $\pi: G \rightarrow$ $\mathrm{GL}(\mathcal{H})$ by bounded operators and norm-continuous unitary representations of $G_{c}$. If $G$ is a finite dimensional semisimple Lie group and $\theta$ a Cartan involution, then this is Weyl's well-known unitary trick relating finite dimensional representations of $G$ to unitary representations of the compact group $G_{c}$.

In the context of the Lüscher-Mack Theorem, it is a natural question which unitary representations of $G_{c}$ are obtained from representations of a semigroup $S_{H}(W)$. To answer this and related questions, we consider for a smooth unitary representation $(\pi, \mathcal{H})$ of a Lie group $G$ the convex function

$$
s_{\pi}: \mathfrak{g} \rightarrow \mathbb{R} \cup\{\infty\}, \quad s_{\pi}(x):=\sup (\operatorname{Spec}(i \mathrm{~d} \pi(x))) .
$$

We call $\pi$ semibounded if $s_{\pi}$ is bounded in the neighborhood of some point $x_{0} \in \mathfrak{g}$, and if $W \subseteq \mathfrak{g}$ is a convex cone, then we say that $\pi$ is $W$-semibounded if $s_{\pi}$ is locally bounded on $W$. Now the converse to the Lüscher-Mack Theorem (Corollary 5.6) asserts that a representation of $G_{c}$ is obtained from a smooth representation of $S$ if and only if it is $i W$-semibounded. Here the main difficulty is to show that, for an $i W$-semibounded unitary representation of $G_{c}$, the prescription $\rho(h \exp x):=$ $\pi(h) e^{-\mathrm{d} \pi(x)}$ define a representation and to see that it is actually smooth. Here we use methods developed previously in [Mer10] and [Ne10b].

With the same tools we obtain the following Holomorphic Extension Theorem (cf. [Ol82] and [Ne00] for the finite dimensional case): For every open invariant cone $W \subseteq \mathfrak{g}$ and a corresponding complex Olshanski semigroup $S_{G}(i W)$, each $W$ semibounded unitary representation of $G$ extends to a holomorphic representation of $S_{G}(i W)$.

In the finite dimensional context Lawson's Theorem guarantees the existence of Olshanski semigroups $S_{H}(W)$ under quite simple requirements on the spectra of the operators ad $x, x \in W$ (cf. [La94], [Ne00]). Since Lawson's arguments involve local compactness in a crucial way, they do not generalize to the Banach context. However, natural examples of Olshanski semigroups arise as compression semigroups of bounded symmetric complex domains in Banach spaces, symmetric Hilbert domains, and real forms of such domains (cf. [Ne01]).

As we show in [MN11], the real forms $\mathcal{D}$ of symmetric Hilbert domains $\mathcal{D}_{c}$, i.e., the fixed points for an antiholomorphic involution $\sigma$, are of particular interest in 
representation theory (see [Kau83, Kau97] for a classification). Here $G_{c}$ is a central extension of the identity component of $\operatorname{Aut}\left(\mathcal{D}_{c}\right)$, which leads to the class of hermitian Lie groups whose semibounded representations are classified in [Ne10c]. Choosing a base point in $\mathcal{D}$ leads to an involution $\theta$ on $G_{c}$ with $\mathcal{D}_{c} \cong G_{c} / G_{c}^{\theta}$, and conjugation with $\sigma$ induces another involution on $G_{c}$. The complex domain $\mathcal{D}_{c}$ has a natural compression semigroup of form $S_{c}=G_{c} \exp \left(W_{c}\right)$, where $W_{c} \subseteq i \mathfrak{g}_{c}$ is an open invariant cone $([\mathrm{Ne} 01])$. The subgroup $H:=\left(G_{c}^{\sigma}\right)_{0}$ is invariant un$\operatorname{der} \theta$ and $\mathcal{D} \cong H / H^{\theta}$ is a real symmetric space. The corresponding semigroup is $S_{H}(W)$ for $W=W_{c}^{\sigma}$. Here our Lüscher-Mack Theorem provides a bridge between *-representations of $S_{H}(W)$ and unitary representations of the group $G_{c}$. A remarkable feature of the infinite dimensional context is that a large class of the irreducible separable continuous unitary representations of $H$ extend to contraction representations of $S_{H}(W)$ with the same commutant and hence further to representations of the larger group $G_{c}$. In particular we obtain an automatic extension of a large class of irreducible representations of $H$ to irreducible semibounded representations of $G_{c}$. For irreducible domains, the irreducible semibounded representations of $G_{c}$ are classified in [Ne10c], and this in turn leads to a classification of the corresponding representations of $H$.

One of the central motivations to study results like the Lüscher-Mack Theorem is that there are natural sources of involutive representations of real Olshanski semigroups $S_{H}(W)$. Here the constructions based on "reflection positivity" are of particular interest because of their connections with euclidean, resp., relativistic quantum field theories (cf. [JO100], [NO11], [LM75], [GJ81]). In this context the semigroup $S_{H}(W)$ constitutes a bridge between unitary representations of the groups $G$ and $G_{c}$. However, these semigroups do not exist in all situations, where the passage from $G$ to $G_{c}$ is of interest, a typical example is the euclidean motion group $G=\mathbb{R}^{4} \rtimes \mathrm{SO}_{4}(\mathbb{R})$ and the Poincaré group $G_{c}=\mathbb{R}^{4} \rtimes \mathrm{SO}_{1,3}(\mathbb{R})$. This was the motivation for Fröhlich, Osterwalder and Seiler to introduce the concept of a virtual representations of a symmetric Lie group ([FOS83]). It would be very interesting to see if a suitable variant of this concept can be developed for Banach-Lie groups.

Notation and conventions. If $\mathcal{H}$ is a Hilbert space, we write $B(\mathcal{H})$ for the algebra of bounded linear operators on $\mathcal{H}, \mathrm{GL}(\mathcal{H})$ for its group of units, and $\mathrm{U}(\mathcal{H}) \subseteq \mathrm{GL}(\mathcal{H})$ for the unitary group.

If $G$ is a topological group and $\mathcal{H}$ a complex Hilbert space, then a unitary representation of $G$ on $\mathcal{H}$, denoted $(\pi, \mathcal{H})$, is a homomorphism $\pi: G \rightarrow \mathrm{U}(\mathcal{H})$ which is continuous with respect to the strong operator topology, i.e., all orbit maps $\pi^{v}: G \rightarrow \mathcal{H}, g \mapsto \pi(g) v$ are continuous.

If $G$ is a Banach-Lie group, then we write $\mathfrak{g}$ for its Lie algebra. It is a Banach-Lie algebra, i.e., a Banach space with a continuous Lie bracket.

If $S$ is a semigroup, we write $\lambda_{s}(t)=s t$ and $\rho_{s}(t)=t s$ for left and right multiplications on $S$. If a neutral element in $S$ exists, it is denoted by $e$.

Let $M$ be a set. A kernel function $K: M \times M \rightarrow \mathbb{C}$ is called positive definite if for $x_{1}, \ldots, x_{n} \in M, n \in \mathbb{N}$, the matrix $\left(K\left(x_{i}, x_{j}\right)\right)_{i, j=1, \ldots n}$ is positive definite. For any such kernel there exists a unique Hilbert subspace $\mathcal{H}_{K} \subseteq \mathbb{C}^{M}$ of functions on $M$ with continuous evaluation maps given by $f(m)=\left\langle f, K_{m}\right\rangle, K_{m}(x)=K(x, m), x, m \in$ $M$. In particular, the subspace $\mathcal{H}_{K}^{0}$, spanned by the functions $K_{m}, m \in M$, is dense in $\mathcal{H}_{K}$. If $\gamma: M \rightarrow \mathcal{H}$ is a function with values in a Hilbert space whose range is total in the sense that it spans a dense subspace and $\langle\gamma(x), \gamma(y)\rangle=K(y, x)$ for $x, y \in M$, then we have a unitary map $\Phi_{\gamma}: \mathcal{H} \rightarrow \mathcal{H}_{K}, \Phi_{\gamma}(v)(x)=\langle v, \gamma(x)\rangle$ (cf. [Ne00, Thm. I.1.6]). 


\section{Geometric Symmetric operators on Reproducing Kernel SPACES}

Let $\mathcal{M}$ be a Banach manifold, $K \in C^{\infty}(\mathcal{M} \times \mathcal{M}, \mathbb{C})$ be a smooth positive definite kernel on $\mathcal{M}$ and $\mathcal{H}_{K} \subseteq \mathbb{C}^{M}$ be the corresponding reproducing kernel Hilbert space. According to [Ne10b, Thm. 7.1], the map $\mathcal{M} \rightarrow \mathcal{H}_{K}, m \mapsto K_{m}$, is smooth, so that $\varphi(m)=\left\langle\varphi, K_{m}\right\rangle$ for $\varphi \in \mathcal{H}_{K}$ implies that $\mathcal{H}_{K} \subseteq C^{\infty}(\mathcal{M}, \mathbb{C})$.

Let $V$ be a vector field on $\mathcal{M}, \mathcal{L}_{V}$ the associated derivation of $C^{\infty}(\mathcal{M}, \mathbb{C})$, and $\varphi_{t}^{V}$ be its local flow, defined at $m \in \mathcal{M}$ for $0 \leq t<\epsilon(m)$. The Lie derivative on functions is given by

$$
\left(\mathcal{L}_{V} \varphi\right)(m):=\left.\frac{d}{d t}\right|_{t=0} \varphi\left(\varphi_{t}^{V} m\right) .
$$

We also consider $\mathcal{L}_{V}$ as an unbounded operator on $\mathcal{H}_{K}$ defined on the domain

$$
\mathcal{D}_{V}:=\left\{\varphi \in \mathcal{H}_{K} \mid \mathcal{L}_{V} \varphi \in \mathcal{H}_{K}\right\}
$$

Definition 2.1. We say that $V$ is symmetric with respect to $K$ (or $K$-symmetric), if

$$
\left(\mathcal{L}_{V} K_{m}\right)(n)=\overline{\left(\mathcal{L}_{V} K_{n}\right)(m)} \text { for } \quad m, n \in \mathcal{M} \text {. }
$$

The key point in the preceding definition is that it can be expressed in terms of the kernel and the vector field, but the following proposition shows that we can draw interesting conclusions for the corresponding unbounded operator on $\mathcal{H}_{K}$.

Proposition 2.2. Let $V$ be a $K$-symmetric vector field. Then, for every $m \in \mathcal{M}$,

$$
\mathcal{L}_{V} K_{m}=\left.\frac{d}{d t}\right|_{t=0} K_{\varphi_{t}^{V} m} \in \mathcal{H}
$$

In particular $\mathcal{H}_{K}^{0} \subseteq \mathcal{D}_{V}$ and the operator $\left.\mathcal{L}_{V}\right|_{\mathcal{H}_{K}^{0}}$ is symmetric. If $\varphi \in \mathcal{H}$ then

$$
\left\langle\varphi, \mathcal{L}_{V} K_{m}\right\rangle=\left(\mathcal{L}_{V} \varphi\right)(m) \quad \text { for } \quad m \in \mathcal{M} .
$$

Proof. Since $\mathcal{M} \rightarrow \mathcal{H}_{K}, m \rightarrow K_{m}$ is smooth, the derivative $\frac{d}{d t} K_{\varphi_{t}^{V} m}$ exists in $\mathcal{H}_{K}$ and, for every $n \in \mathcal{M}$,

$$
\left(\mathcal{L}_{V} K_{m}\right)(n)=\overline{\left(\mathcal{L}_{V} K_{n}\right)(m)}=\left.\frac{d}{d t}\right|_{t=0} K\left(n, \varphi_{t}^{V} m\right)=\left(\left.\frac{d}{d t}\right|_{t=0} K_{\varphi_{t}^{V} m}\right)(n) .
$$

For every $\varphi \in \mathcal{H}$ we therefore have

$$
\left\langle\varphi, \mathcal{L}_{V} K_{m}\right\rangle=\left\langle\varphi,\left.\frac{d}{d t}\right|_{t=0} K_{\varphi_{t}^{V} m}\right\rangle=\left.\frac{d}{d t}\right|_{t=0} \varphi\left(\varphi_{t}^{V} m\right)=\left(\mathcal{L}_{V} \varphi\right)(m)
$$

Remark 2.3. Note that (3) implies in particular

$$
\left\langle\varphi, \mathcal{L}_{V} K_{m}\right\rangle=\left\langle\mathcal{L}_{V} \varphi, K_{m}\right\rangle \quad \text { for } \quad \varphi \in \mathcal{D}_{V}
$$

Below we recall Fröhlich's Theorem on unbounded symmetric semigroups as it is stated in [Frö80, Cor. 1.2]. Actually Fröhlich assumes that the Hilbert space $\mathcal{H}$ is separable, but this is not necessary for the conclusion. Replacing the assumption of weak measurability by weak continuity, all arguments in [Frö80] work for nonseparable spaces as well.

Theorem 2.4 (Fröhlich). Let $H$ be a symmetric operator defined on the domain $\mathcal{D}$ dense in the Hilbert space $\mathcal{H}$. Suppose that for every $\Phi \in \mathcal{D}$ there exists $\epsilon(\Phi)>0$ such that the equation

$$
\frac{d}{d t} \Phi(t)=H \Phi(t)
$$

has a solution satisfying $\lim _{t \rightarrow 0} \Phi(t)=\Phi$ and $\Phi(t) \in \mathcal{D}$ for $0 \leq t<\epsilon(\Phi)$. Then the operator $H$ is essentially self-adjoint and $\Phi(t)=e^{t \bar{H}} \Phi$. 
We apply Fröhlich's Theorem to our geometric operator $\mathcal{L}_{V}$ from Proposition 2.2. The new feature is that we can even describe the closure of the essentially selfadjoint operator we obtain.

Theorem 2.5 (Geometric Fröhlich Theorem). Let $\mathcal{M}$ be a Banach manifold and $K$ be a smooth positive definite kernel. Then $\mathcal{H}_{K}$ consists of smooth functions, and if $V$ is a $K$-symmetric vector field on $\mathcal{M}$, then the Lie derivative $\mathcal{L}_{V}$ defines an essentially self-adjoint operator $\mathcal{H}_{K}^{0} \rightarrow \mathcal{H}_{K}$ whose closure $\mathcal{L}_{V}^{K}$ coincides with $\left.\mathcal{L}_{V}\right|_{\mathcal{D}_{V}}$. Moreover, if the local flow $\Phi_{t}^{V}(m)$ of $m \in \mathcal{M}$ at time $t$ is defined, then

$$
e^{t \mathcal{L}_{V}^{K}} K_{m}=K_{\Phi_{t}^{V}(m)} .
$$

Proof. We apply Fröhlich's Theorem with $\mathcal{D}=\mathcal{H}_{K}^{0}$ and $H=\left.\mathcal{L}_{V}\right|_{\mathcal{D}}$. For $\Phi=$ $\sum_{j=1}^{k} \alpha_{j} K_{m_{j}}, \alpha_{j} \in \mathbb{C}, m_{j} \in M$, we define

$$
\Phi(t)=\sum_{j=1}^{k} \alpha_{j} K_{\varphi_{t}^{V} m_{j}}, \quad \text { for } \quad t<\min \left(\epsilon\left(m_{1}\right), \ldots, \epsilon\left(m_{k}\right)\right) .
$$

Then (2) implies $\frac{d}{d t} \Phi(t)=\mathcal{L}_{V} \Phi(t)$, so the assumptions of Fröhlich's Theorem are satisfied, and $\left.\mathcal{L}_{V}\right|_{\mathcal{H}_{K}^{0}}$ is essentially self-adjoint. Let us denote by $T_{V}$ its closure. If $\varphi$ is an element of its domain $\mathcal{D}\left(T_{V}\right)$, then (3) leads for every $m \in \mathcal{M}$ to

$$
\left(T_{V} \varphi\right)(m)=\left\langle T_{V} \varphi, K_{m}\right\rangle=\left\langle\varphi, \mathcal{L}_{V} K_{m}\right\rangle=\left(\mathcal{L}_{V} \varphi\right)(m) .
$$

We conclude that $\mathcal{D}\left(T_{V}\right) \subseteq \mathcal{D}_{V}$ and $T_{V}=\left.\mathcal{L}_{V}\right|_{\mathcal{D}\left(T_{V}\right)}$. The other inclusion follows from (4) which implies that $\mathcal{D}_{V} \subseteq \mathcal{D}\left(\left(\left.\mathcal{L}_{V}\right|_{\mathcal{H}_{K}^{0}}\right)^{*}\right)$. Indeed, since $\left.\mathcal{L}_{V}\right|_{\mathcal{H}_{K}^{0}}$ is essentially self-adjoint we have $\left(\left.\mathcal{L}_{V}\right|_{\mathcal{H}_{K}^{0}}\right)^{*}=T_{V}$.

Remark 2.6. Theorem 2.4 above extends directly to the case where $\mathcal{M}$ is a manifold modeled on a locally convex space, provided the vector field $V$ is assumed to have a local flow. Indeed [Ne10b, Thm. 7.1] is stated in this generality.

\section{The LÜscher-Mack Theorem}

Let $(G, \theta)$ be a symmetric Banach-Lie group. We also write $\theta$ for the corresponding automorphism of its Lie algebra $\mathfrak{g}$, which leads to the symmetric Banach-Lie algebra $(\mathfrak{g}, \theta)$. We write

$$
\mathfrak{g}=\mathfrak{h} \oplus \mathfrak{q} \quad \text { with } \quad \mathfrak{h}=\operatorname{ker}(\theta-\mathbf{1}) \quad \text { and } \quad \mathfrak{q}=\operatorname{ker}(\theta+\mathbf{1}),
$$

for the eigenspace decomposition of $\mathfrak{g}$ under $\theta$. Let $\emptyset \neq W \subseteq \mathfrak{q}$ be an open convex cone invariant under $e^{\text {ad } \mathfrak{h}}$ and $S=S_{H}(W)$ be a corresponding Olshanski semigroup in the sense of Definition A.4. In particular, $H$ is a connected Lie group with Lie algebra $\mathfrak{h}$, but we do not assume that $H$ is contained in $G$. We write

$$
\exp : W \rightarrow S=S_{H}(W)
$$

for the corresponding exponential map on $W$. In polar coordinates the involution on $S$ is given by

$$
(h \exp x)^{*}=(\exp x) h^{-1}=h^{-1} \exp (\operatorname{Ad}(h) x)
$$

(Remark A.5).

Examples 3.1. (a) Let $\mathcal{H}$ be a real Hilbert space and $G=\operatorname{GL}(\mathcal{H})_{0}$. We write $A^{\top}$ for the adjoint of an element $A \in B(\mathcal{H})$. Then $\theta(g)=\left(g^{\top}\right)^{-1}$ defines an involution on $G$ with $H=G^{\theta}=\mathrm{O}(\mathcal{H})_{0}$ (the orthogonal group of $\mathcal{H}$ ) and $\mathfrak{q}=\operatorname{Sym}(\mathcal{H})$ is the space of symmetric operators. In this case $G=\mathrm{O}(\mathcal{H})_{0} \exp (\operatorname{Sym}(\mathcal{H}))$ actually is an Olshanski (semi-)group for $W=\mathfrak{q}=\operatorname{Sym}(\mathcal{H})$. Similarly statements hold for complex and quaternionic Hilbert spaces. 
(b) If $H$ is a connected Lie group for which $\operatorname{Ad}(H)$ leaves a compatible norm on $\mathfrak{h}$ invariant, then $\mathfrak{h}$ is called an elliptic Banach-Lie algebra. Then $H$ has a universal complexification $\eta: H \rightarrow H_{\mathbb{C}}$ with a polar decomposition $H_{\mathbb{C}}=H \exp (i \mathfrak{h})([\mathrm{Ne} 02$, Ex. 6.9]). A finite dimensional Lie algebra $\mathfrak{h}$ is elliptic if and only if it is compact, but the class of elliptic Lie algebras is quite large. In particular, it contains the algebra $\mathfrak{u}(\mathcal{A})$ of skew-hermitian elements of a $C^{*}$-algebra $\mathcal{A}$ and in particular the Lie algebra $\mathfrak{u}(\mathcal{H})$ of the full unitary group $\mathrm{U}(\mathcal{H})$ of a complex Hilbert space $\mathcal{H}$.

(c) If $V$ is a Banach space and $W \subseteq V$ an open convex cone, then $S=S_{V}(W)=$ $V+i W \subseteq V_{\mathbb{C}}$ is a complex Olshanski semigroup with respect to the involution $(x+i y)^{*}:=-x+i y$.

(d) If $\mathcal{A}$ is a unital $C^{*}$-algebra, $G:=\mathcal{A}_{0}^{\times}$(the identity component of its group of units) and $S:=\{s \in G:\|s\|<1\}$, then $S$ is a complex Olshanski semigroup $S=S_{\mathrm{U}(\mathcal{A})_{0}}(W)$, where

$$
W=\left\{x \in \mathcal{A}: x^{*}=x, \sup (\operatorname{Spec}(x))<0\right\} .
$$

An important example is the semigroup of invertible strict contractions of a complex Hilbert space $\mathcal{H}$.

(e) Let $\mathcal{A}$ be a unital $C^{*}$-algebra and $\tau=\tau^{2}=\tau^{*} \in \mathcal{A}$. For $a, b \in \mathcal{A}$, we write $a<b$ if there exists an invertible element $c \in \mathcal{A}$ with $b-a=c^{*} c$. Then

$$
S:=\left\{s \in \mathcal{A}^{\times}: s^{*} \tau s<\tau\right\}
$$

is an open subsemigroup of $\mathcal{A}$ with respect to multiplication. To see that it is non-empty, we observe that we may write $\tau=\mathbf{1}-2 p=(\mathbf{1}-p)-p$ for a projection $p=p^{*}=p^{2} \in \mathcal{A}$. For $\lambda \in \mathbb{C}^{\times}$and $s:=\lambda(\mathbf{1}-p)+\lambda^{-1} p$ we then have

$$
s^{*} \tau s=|\lambda|^{2}(\mathbf{1}-p)-\left|\lambda^{-1}\right|^{2} p<\tau=(\mathbf{1}-p)-p
$$

if and only if $|\lambda|<1$. The semigroup $S$ is of the form $S_{H}(W)$ for

$$
H=\left\{g \in \mathcal{A}^{\times}: g^{*} \tau g=\tau\right\} .
$$

(f) As already mentioned in the introduction, the compression semigroups of (real forms of) symmetric (Hilbert) domains are also Olshanski semigroups (cf. [Ne01]).

Definition 3.2. Let $(S, *)$ be an involutive Banach semigroup, i.e., an involutive semigroup carrying a Banach manifold structure such that multiplication and inversion are smooth maps.

(a) A homomorphism $\rho: S \rightarrow B(\mathcal{H})$ is called a $*$-representation if $\rho\left(s^{*}\right)=\rho(s)^{*}$ for every $s \in S$. Such a representation is said to be non-degenerate if $\rho(S) \mathcal{H}$ spans a dense subspace of $\mathcal{H}$, which is equivalent to the condition that $\rho(S) v=\{0\}$ implies $v=0$.

(b) For a representation $(\rho, \mathcal{H})$ of $S$, a vector $v \in \mathcal{H}$ is called smooth if its orbit map $\rho^{v}: S \rightarrow \mathcal{H}, s \mapsto \rho(s) v$ is smooth. We write $\mathcal{H}^{\infty}$ for the subspace of smooth vectors and say that $(\rho, \mathcal{H})$ is smooth if $\mathcal{H}^{\infty}$ is dense in $\mathcal{H}$.

(c) A *-representation $(\rho, \mathcal{H})$ of $S$ is called locally bounded if every $s \in S$ has a neighborhood on which $\|\rho(\cdot)\|$ is bounded.

(d) For a unitary representation $(\pi, \mathcal{H})$ of a Lie group $G$, we also write $\mathcal{H}^{\infty}:=$ $\mathcal{H}^{\infty}(\pi)$ for the subspace of smooth vectors. This subspace carries the derived representation

$$
\mathrm{d} \pi: \mathfrak{g} \rightarrow \operatorname{End}\left(\mathcal{H}^{\infty}\right), \quad \mathrm{d} \pi(x) v:=\left.\frac{d}{d t}\right|_{t=0} \pi(\exp t x) v
$$

of the Lie algebra $\mathfrak{g}$ of $G$. If $\pi$ is smooth, then these operators are essentially skewadjoint and, for $x \in \mathfrak{g}$, the closure $\overline{\mathrm{d} \pi}(x)$ is the infinitesimal generator of the unitary one-parameter group $\pi(\exp t x)$. 
Remark 3.3. (a) Since the arguments in [Ne10b, Prop. 5.1, Lemma 5.2] apply also to semigroup actions, the first countability of $S$ implies that any strongly continuous representation $\rho: S \rightarrow B(\mathcal{H})$ defines a continuous action $S \times \mathcal{H} \rightarrow \mathcal{H}$. The continuity in the points $(s, 0)$ implies that $\rho$ is locally bounded.

(b) If, conversely, $\rho$ is locally bounded and the orbit maps $\rho^{v}: S \rightarrow \mathcal{H}$ are continuous for every $v$ in a dense subspace, then $\rho$ is strongly continuous ([Ne00, Lemma IV.1.3]).

In the following we are interested in non-degenerate strongly continuous $*-$ representations $\rho: S=S_{H}(W) \rightarrow B(\mathcal{H})$ on a Hilbert space $\mathcal{H}$. First we observe that, although $H$ need not be contained in $S$, any non-degenerate $*$-representation of $S$ defines in a natural fashion a unitary representation of $H$.

Proposition 3.4. For every non-degenerate $*$-representation $(\rho, \mathcal{H})$ of $S$ there exists a unique, not necessarily continuous, unitary representation $\rho_{H}: H \rightarrow \mathrm{U}(\mathcal{H})$ satisfying

$$
\rho(h s)=\rho_{H}(h) \rho(s) \quad \text { for } \quad h \in H, s \in S .
$$

Its space of continuous vectors contains $\rho(S) \mathcal{H}$ and its space of smooth vectors contains $\rho(S) \mathcal{H}^{\infty}$. In particular, $\left(\rho_{H}, \mathcal{H}\right)$ is strongly continuous, resp., smooth if $(\rho, \mathcal{H})$ is.

Proof. The existence of a unique homomorphism $\rho_{H}: H \rightarrow \mathrm{U}(\mathcal{H})$ satisfying (6) follows from the fact that $H$ acts on $S$ by unitary multipliers (Remark A.5, [Ne00, Rem. III.1.5]). The smoothness of the action of $H$ on $S$ now implies that $\rho(s) v$ has a continuous (smooth) orbit map under $H$ if $v$ has a continuous (smooth) orbit map under $S$. This completes the proof.

To apply the Hille-Yosida Theorem to the symmetric one-parameter semigroups $\rho_{x}(t):=\rho(\exp t x)$ for $x \in W$, we need to know that

$$
\lim _{t \rightarrow 0} \rho_{x}(t) v=v \text { for } v \in \mathcal{H} .
$$

As the following lemma shows, this follows from the non-degeneracy of the representation and strong continuity.

Lemma 3.5. If $\rho$ is a non-degenerate $*$-representation of $S=S_{H}(W)$ and $x \in W$, then $\rho(\exp x) \mathcal{H}$ is dense in $\mathcal{H}$. Furthermore, (7) holds if $\rho_{x}$ is strongly continuous on $\mathbb{R}_{>0}$.

Proof. Let $v \in(\rho(\exp x) \mathcal{H})^{\perp}$. In view of

$$
\langle\rho(\exp x) w, v\rangle=\langle w, \rho(\exp x) v\rangle,
$$

this is equivalent to $\rho(\exp x) v=0$. Now [Ne00, Cor. II.4.15] implies that

$$
\rho\left(\exp \frac{x}{n}\right) v=0 \quad \text { for every } \quad n>0
$$

and hence that $\rho(\exp t x) v=0$ for every $t>0$.

Any $s \in S$ can be written $s=s_{0} \exp (t x)$ with $s_{0} \in S$ and some $t>0$ because the left invariant vector field $V_{x}$ generates a local flow on $S$ (Remark A.9). It follows that $\rho(s) v=\rho\left(s_{0}\right) \rho(\exp t x) v=0$. Since $\rho$ is a non-degenerate representation of $S$, it follows that $v=0$, and hence that $\rho(\exp x) \mathcal{H}$ is dense in $\mathcal{H}$.

We see in particular, that the representation $\rho_{x}$ of $\mathbb{R}_{>0}$ is non-degenerate. Assume that $\rho_{x}$ is strongly continuous, hence locally bounded (Remark 3.3). Then Lemma [Ne00, Lemma VI.2.2] implies that $\rho_{x}$ extends uniquely to a strongly continuous representation on $\mathbb{R}_{\geq 0}$, which implies (7). 
Definition 3.6. Let $(\rho, \mathcal{H})$ be a non-degenerate strongly continuous $*$-representation of $S$. In view of Lemma 3.5, we obtain for every $x \in W$ a self-adjoint operator

$$
\overline{\mathrm{d} \rho}(x) \xi:=\left.\frac{d}{d t}\right|_{t=0} \rho(\exp t x) \xi
$$

the generator of the symmetric one-parameter semigroup $\rho_{x}$. It is defined on the subspace $\mathcal{D}(\overline{\mathrm{d} \rho}(x))$ where the derivative exists (Hille-Yosida Theorem). For $x \in \mathfrak{h}$, we likewise write $\overline{\mathrm{d} \rho}(x)$ for the generator of the corresponding strongly continuous unitary one-parameter group $\rho_{x}(t):=\rho_{H}(\exp t x)$ (Proposition 3.4; Stone's Theorem).

The following theorem is our main result.

Theorem 3.7. Let $G_{c}$ be a simply connected Lie group with Lie algebra

$$
\mathfrak{g}_{c}=\mathfrak{h} \oplus i \mathfrak{q} \subseteq \mathfrak{g}_{\mathbb{C}},
$$

and $\rho: S=S_{H}(W) \rightarrow B(\mathcal{H})$ be a non-degenerate strongly continuous smooth $*-$ representation. Then there exists a unique smooth unitary representation $(\pi, \mathcal{H})$ of $G_{c}$ on $\mathcal{H}$ whose space of smooth vectors is contained in $\mathcal{D}(\overline{\mathrm{d} \rho}(x))$ for every $x \in \mathfrak{h} \cup W$, and whose derived representation satisfies

$$
\mathrm{d} \pi(x+i y) \subseteq \overline{\mathrm{d} \rho}(x)+i \overline{\mathrm{d} \rho}(y) \text { for } x \in \mathfrak{h} \text { and } y \in W .
$$

We will see (Remark 4.10) that the space $\mathcal{H}^{\infty}(\pi)$ of smooth vectors for the representation $\pi$ of $G_{c}$ coincides with

$$
\left.\bigcap_{x_{j} \in \mathfrak{h} \cup W, n \in \mathbb{N}} \mathcal{D}\left(\overline{\mathrm{d} \rho}\left(x_{n}\right)\right) \ldots \overline{\mathrm{d} \rho}\left(x_{1}\right)\right) .
$$

Accordingly, our strategy is to define a representation of the Lie algebra $\mathfrak{g}_{c}$ on this space so that (8) is satisfies and then verify that we can use the results in [Mer10] to show that this representation of $\mathfrak{g}_{c}$ integrates to a representation of $G_{c}$.

Remark 3.8. (a) Let $q_{H}: \widetilde{H} \rightarrow H$ be the simply connected covering group of $H$. Then we have a unique morphism $\iota: \widetilde{H} \rightarrow G_{c}$ integrating the inclusion map $\mathfrak{h} \rightarrow \mathfrak{g}_{c}$. The relation $\pi \circ \iota=\rho_{H} \circ q_{H}$ now implies that $\iota\left(\operatorname{ker} q_{H}\right) \subseteq \operatorname{ker} \pi$. As $\operatorname{ker} q_{H}$ acts trivially on $\mathfrak{g}$, it also acts trivially on $\mathfrak{g}_{c}$, i.e., $\iota\left(\operatorname{ker} q_{H}\right) \subseteq Z\left(G_{c}\right)$. In particular, it is a normal subgroup and $\pi$ actually factors through a representation of the quotient $G_{c} / \iota\left(\operatorname{ker} q_{H}\right)$. If $\iota\left(\operatorname{ker} q_{H}\right)$ is discrete, this quotient is a Lie group with the same Lie algebra.

Here is an example showing that the group $\iota\left(\operatorname{ker} q_{H}\right)$ need not be discrete. We consider the Lie algebra $\mathfrak{g}=\mathfrak{s l}_{2}(\mathbb{R}) \oplus \mathbb{R}$ with the involution $\theta(x, t)=\left(-x^{\top}, t\right)$, which leads to $\mathfrak{g}_{c}=\mathfrak{s u}_{2}(\mathbb{C}) \oplus \mathbb{R}$, and $G_{c}:=\mathrm{SU}_{2}(\mathbb{C}) \times \mathbb{R}$ is a corresponding simply connected group.

For the Lie algebra $\mathfrak{h}=\mathfrak{g}^{\theta}=\mathfrak{s o}_{2}(\mathbb{R}) \oplus \mathbb{R}$, the corresponding simply connected group is $\widetilde{H}=\mathbb{R}^{2}$, and the kernel for the adjoint action of $\widetilde{H}$ on $\mathfrak{g}$ is isomorphic to $\mathbb{Z} \times \mathbb{R}$ with $\operatorname{ker} \iota=2 \mathbb{Z} \times\{0\}$. Now $\Gamma:=\mathbb{Z}(2,1) \oplus \mathbb{Z}(0, \sqrt{2})$ is a discrete subgroup of $\widetilde{H}$ acting trivially on $\mathfrak{g}$, so that $H:=\widetilde{H} / \Gamma$ satisfies our condition imposed for the construction of Olshanski semigroups and $\operatorname{ker} q_{H}=\Gamma$. Now $\iota(\Gamma)=\{\mathbf{1}\} \times(\mathbb{Z}+\sqrt{2} \mathbb{Z})$ is not discrete in $G_{c}$.

(b) Let $q_{S}: \widetilde{S}=S_{\widetilde{H}}(W)$ be the universal covering of the Olshanski semigroup $S=S_{H}(W)$ and $(\rho, \mathcal{H})$ be a smooth locally bounded *-representation of $S$, so that Theorem 3.7 leads to a unitary representation $(\pi, \mathcal{H})$ of $G_{c}$. Clearly, the representation $\widetilde{\rho}:=\rho \circ q_{S}$ of $\widetilde{S}$ leads to the same representation of $G_{c}$.

Now $\pi \circ \iota=\widetilde{\rho}_{H}$ implies that $\operatorname{ker}\left(\widetilde{\rho}_{H}\right) \supseteq \operatorname{ker} \iota$, so that the representation $\widetilde{\rho}$ of $\widetilde{S}$ actually factors through a representation of the semigroup $S_{c}:=S_{H_{c}}(W)$, where $H_{c}=\iota(\widetilde{H})$. From the point of view of the representation theory of the group $G_{c}$, all 
representations obtained by Theorem 3.7 can also be obtained from the semigroup $S_{c}$, which is locally isomorphic to $S$.

The representation $(\pi, \mathcal{H})$ of $G_{c}$ obtained from the Lüscher-Mack Theorem has the remarkable property that the spectrum of the operator $i \overline{\mathrm{d}} \pi(x)$ is bounded from below for every $x$ in the cone $i W \subseteq \mathfrak{g}_{c}$. In Section 5 we will prove a converse to Theorem 3.7: A $-i W$-semibounded representation $\pi$ of $G_{c}$ comes from a strongly continuous smooth $*$-representation of $S_{H}(W)$, where $H=\left\langle\exp _{G_{c}} \mathfrak{h}\right\rangle$ is the identity component of the group of fixed points for the involution $\theta_{c}$ on $G_{c}$ acting on the Lie algebra by $x+i y \mapsto x-i y$ for $x \in \mathfrak{h}, y \in \mathfrak{q}$.

Remark 3.9. It is instructive to take a closer look at the special case $\mathfrak{g}=\mathfrak{h}_{\mathbb{C}}$ with $\mathfrak{q}=i \mathfrak{h}$, i.e., where $\theta(z)=\bar{z}$ is complex conjugation with respect to the real form $\mathfrak{h}$. Then $S_{H}(W)$ is a complex Olshanski semigroup (cf. Definition A.4(b)). Then the complexification of $\mathfrak{g}$ can be realized by the embedding

$$
\eta: \mathfrak{g} \rightarrow \mathfrak{g} \oplus \mathfrak{g}, \quad z \mapsto(z, \bar{z}),
$$

which leads to $\mathfrak{g}_{\mathbb{C}} \cong \mathfrak{g} \oplus \mathfrak{g}$, a direct sum of two complex Lie algebras. In this picture the Lie algebra $\mathfrak{g}_{c}=\mathfrak{h}+i \mathfrak{q}$ is given by

$$
\{(x, x): x \in \mathfrak{h}\}+i\{(y,-y): y \in i \mathfrak{h}\}=\{(x, x): x \in \mathfrak{h}\}+\{(y,-y): y \in \mathfrak{h}\}=\mathfrak{h} \oplus \mathfrak{h} .
$$

We conclude that $G_{c} \cong \widetilde{H} \times \widetilde{H}$, where $\widetilde{H}$ is the simply connected covering group of $H$.

If $\alpha: \mathfrak{g} \rightarrow \operatorname{End}(\mathcal{D})$ is a complex linear representation of $\mathfrak{g}$ on the complex linear space $\mathcal{D}$, then the complex linear extension to $\mathfrak{g}_{\mathbb{C}} \cong \mathfrak{g} \oplus \mathfrak{g}$ is given by

$$
\alpha_{\mathbb{C}}(z, w)=\frac{1}{2}(\alpha(z+\bar{w})+i \alpha(i \bar{w}-i z))=\alpha(z)
$$

because

$$
(z, w)=\frac{1}{2}((z+\bar{w}, \bar{z}+w)+i(i \bar{w}-i z, \overline{i \bar{w}-i z})) .
$$

Therefore the corresponding unitary representation $\pi_{c}$ of $G_{c}$ is given by $\pi_{c}\left(h_{1}, h_{2}\right)=$ $\rho_{H}\left(h_{1}\right)$.

\section{Proof of the Lüscher-Mack Theorem}

The following lemma permits us to reduce the proof of Theorem 3.7 to the case where $(\rho, \mathcal{H})$ is cyclic and generated by a smooth vector.

Lemma 4.1. A non-degenerate strongly continuous smooth *-representation is a direct sum of cyclic representations with smooth cyclic vectors.

Proof. The set of all families $\left(\mathcal{H}_{j}\right)_{j \in J}$ of mutually orthogonal closed $S$-invariant subspaces which contain a smooth cyclic vector is well ordered by inclusion and by Zorn's Lemma it has a maximal element $\left(\mathcal{H}_{j}\right)_{j \in J}$. Let $\mathcal{K}=\overline{\bigoplus_{j \in J} \mathcal{H}_{j}}$. Then $\mathcal{K}$ and $\mathcal{K}^{\perp}$ are $S$-invariant, and we claim that if $\mathcal{K}^{\perp}$ is non-zero, it contains a smooth vector. Indeed, if $\mathcal{K} \neq \mathcal{H}$, there exists a smooth vector $w$ which is not in $\mathcal{K}$. Let us write pr for the orthogonal projection on $\mathcal{K}^{\perp}$ and $v:=\operatorname{pr}(w)$. Then the relation $\rho(s) v=\operatorname{pr}(\rho(s) w), s \in S$, shows that $v$ is a smooth vector in $\mathcal{K}^{\perp}$. Since $\rho$ is non-degenerate, we have $v \in \overline{\rho(S) v}$ (cf. [Ne00, Lemma II.2.4]), hence $\overline{\operatorname{span} \rho(S) v}$ is a closed $S$-invariant subspace with a smooth cyclic vector orthogonal to each $\mathcal{H}_{j}$, $j \in J$. But this contradicts the maximality of $\left(\mathcal{H}_{j}\right)_{j \in J}$. Therefore $\mathcal{H}=\mathcal{K}$, and as representations of $S$, we have $\mathcal{H} \simeq \widehat{\bigoplus}_{j \in J} \mathcal{H}_{j}$.

From now on we assume that $v_{0} \in \mathcal{H}^{\infty}$ is a cyclic vector. Then we obtain a smooth positive definite kernel

$$
K\left(s_{1}, s_{2}\right):=K_{s_{2}}\left(s_{1}\right):=\left\langle\rho\left(s_{1} s_{2}^{*}\right) v_{0}, v_{0}\right\rangle
$$


on $S$ which leads to a unitary map

$$
\Psi: \mathcal{H} \rightarrow \mathcal{H}_{K}, \quad \Psi(v)(s):=\rho^{v, v_{0}}(s)=\left\langle\rho(s) v, v_{0}\right\rangle=\left\langle v, \rho\left(s^{*}\right) v_{0}\right\rangle
$$

onto the reproducing kernel space $\mathcal{H}_{K} \subseteq C^{\infty}(S, \mathbb{C})$. Indeed, $\Psi$ intertwines $\rho$ with the representation of $S$ on $\mathcal{H}_{K} \subseteq C^{\infty}(S, \mathbb{C})$ given by $\left(\rho\left(s_{2}\right) \varphi\right)\left(s_{1}\right)=\varphi\left(s_{1} s_{2}\right)$. Therefore it suffices to prove Theorem 3.7 only for the representation $\left(\rho, \mathcal{H}_{K}\right)$ of $S=S_{H}(W)$.

The function $\varphi_{0}=\rho^{v_{0}, v_{0}}=\Psi\left(v_{0}\right)$ satisfies $\rho(s) \varphi_{0}=K_{s^{*}}$, so that

$$
\mathcal{H}_{K}^{0}=\operatorname{span}\left(\rho(S) \varphi_{0}\right) \subseteq \mathcal{H}_{K}^{\infty} \quad \text { and } \quad \rho\left(s_{2}\right) K_{s_{1}}=K_{s_{1} s_{2}^{*}}
$$

for $s_{1} \in S, s_{2} \in S \cup H$. To each $x \in \mathfrak{g}$ we associate the corresponding left invariant vector field $V_{x}$ on $S$ (cf. Definition A.8) and write the corresponding integral curves as $s \exp (t x)$ (cf. Remark A.9). As in (1), we set

$$
\mathcal{L}_{x}:=\mathcal{L}_{V_{x}} \quad \text { and } \quad \mathcal{L}_{x}^{K}:=\left.\mathcal{L}_{x}\right|_{\mathcal{D}_{x}} \quad \text { for } \mathcal{D}_{x}:=\mathcal{D}_{V_{x}}=\left\{\varphi \in \mathcal{H}_{K}: \mathcal{L}_{x} \varphi \in \mathcal{H}_{K}\right\},
$$

and extend this definition $\mathbb{C}$-linearly to any $x \in \mathfrak{g}_{\mathbb{C}}$. Then we have on $C^{\infty}(S, \mathbb{C})$ the relation

$$
\left[\mathcal{L}_{x}, \mathcal{L}_{y}\right]=\mathcal{L}_{[x, y]}
$$

We recall that a core of a self-adjoint operator $A: \mathcal{D} \rightarrow \mathcal{H}$ is a dense subspace $\mathcal{D}_{0} \subseteq \mathcal{D}$ for which $A=\overline{\left.A\right|_{\mathcal{D}_{0}}}$.

Proposition 4.2. Let $x \in \mathfrak{h} \cup W$. Then $\mathcal{H}_{K}^{0} \subseteq \mathcal{D}(\overline{\mathrm{d} \rho}(x))$, $\mathcal{H}_{K}^{0}$ is a core for $\overline{\mathrm{d} \rho}(x)$, and

$$
\overline{\mathrm{d} \rho}(x)=\mathcal{L}_{x}^{K}
$$

Proof. For $x \in \mathfrak{h}$, the first assertion follows from (9) and Proposition 3.4. For $x \in W$ and $s \in S$, the map $t \mapsto \rho(\exp (t x) s) \varphi_{0}$ extends to a smooth map on some 0 -neighborhood, and for $t \geq 0$ it coincides with $e^{t \overline{\mathrm{d} \rho}(x)} \rho(s) \varphi_{0}$. Therefore $\rho(s) \varphi_{0}$ is contained in the domain $\mathcal{D}(\overline{\mathrm{d} \rho}(x))$ of $\overline{\mathrm{d} \rho}(x)$.

In the following we also write $\rho(h):=\rho_{H}(h)$ for $h \in H$. For $x \in \mathfrak{h} \cup W$, the space $\mathcal{H}_{K}^{0}$ is invariant under $\rho(\exp t x), t>0$, hence is a core for $\overline{\mathrm{d} \rho}(x)$ ([EN00, Prop. 1.7]), i.e., $\overline{\mathrm{d} \rho}(x)=\overline{\left.\overline{\mathrm{d} \rho}(x)\right|_{\mathcal{H}_{K}^{0}}}$.

Finally, let $x \in \mathfrak{h} \cup W$ and $\varphi \in \mathcal{D}(\overline{\mathrm{d} \rho}(x))$. Then

$$
\begin{aligned}
& (\overline{\mathrm{d} \rho}(x) \varphi)(s)=\left\langle\left.\frac{d}{d t}\right|_{t=0} \rho(\exp t x) \varphi, K_{s}\right\rangle=\left.\frac{d}{d t}\right|_{t=0}\left\langle\varphi, \rho\left((\exp t x)^{*}\right) K_{s}\right\rangle \\
& =\left.\frac{d}{d t}\right|_{t=0}\left\langle\varphi, K_{s \exp t x}\right\rangle=\left.\frac{d}{d t}\right|_{t=0} \varphi(s \exp t x)=\left(\mathcal{L}_{x} \varphi\right)(s),
\end{aligned}
$$

shows that $\mathcal{L}_{x}$ extends $\overline{\mathrm{d} \rho}(x)$. Further, for $\psi \in \mathcal{D}_{x}$,

$$
\begin{aligned}
\left\langle\mathcal{L}_{x} \psi, K_{s}\right\rangle & =\left(\mathcal{L}_{x} \psi\right)(s)=\left.\frac{d}{d t}\right|_{t=0} \psi(s \exp (t x))=\left\langle\psi,\left.\frac{d}{d t}\right|_{t=0} K_{s \exp t x}\right\rangle \\
& =\left\langle\psi,\left.\frac{d}{d t}\right|_{t=0} \rho(\exp t x)^{*} K_{s}\right\rangle=\left\langle\psi, \overline{\mathrm{d} \rho}(-\theta(x)) K_{s}\right\rangle .
\end{aligned}
$$

shows that $\left(\left.\overline{\mathrm{d} \rho}(-\theta(x))\right|_{\mathcal{H}_{K}^{0}}\right)^{*}=\overline{\mathrm{d} \rho}(x)$ extends $\mathcal{L}_{x}$. This proves $(11)$.

The preceding proposition shows that, for any $x \in \mathfrak{h} \cup W, \mathcal{L}_{x}$ leaves the subspace

$$
\begin{aligned}
\mathcal{D} & :=\bigcap_{n \in \mathbb{N}, x_{n}, \ldots, x_{1} \in \mathfrak{h} \cup W} \mathcal{D}\left(\overline{\mathrm{d} \rho}\left(x_{n}\right) \ldots \overline{\mathrm{d} \rho}\left(x_{1}\right)\right) \\
& =\left\{\varphi \in \mathcal{H}_{K}:(\forall n \in \mathbb{N})\left(\forall x_{n}, \ldots, x_{1} \in \mathfrak{h} \cup W\right) \mathcal{L}_{x_{1}} \cdots \mathcal{L}_{x_{n}} \varphi \in \mathcal{H}_{K}\right\}
\end{aligned}
$$

invariant, and by linearity this is also true for $x \in \mathfrak{g}_{\mathbb{C}}$. Therefore we set

$$
\alpha(x):=\left.\mathcal{L}_{x}\right|_{\mathcal{D}} \quad \text { for } \quad x \in \mathfrak{g}_{\mathbb{C}}
$$


and observe that

$$
\mathcal{D}=\left\{\varphi \in \mathcal{H}_{K}:(\forall n \in \mathbb{N})\left(\forall x_{1}, \ldots, x_{n} \in \mathfrak{g}\right) \mathcal{L}_{x_{1}} \cdots \mathcal{L}_{x_{n}} \varphi \in \mathcal{H}_{K}\right\} .
$$

Lemma 4.3. If $x_{1}, \ldots x_{n} \in \mathfrak{g}, n \in \mathbb{N}$, then

$$
\mathcal{L}_{x_{n}} \ldots \mathcal{L}_{x_{1}} \mathcal{H}_{K}^{0} \subseteq \mathcal{H}_{K}
$$

and for $s \in S$,

$$
\mathcal{L}_{x_{n}} \ldots \mathcal{L}_{x_{1}} \rho(s) \varphi_{0}=\left.\frac{\partial t^{n}}{\partial t_{n} \ldots \partial t_{1}}\right|_{t_{n}=\cdots=t_{1}=0} \rho\left(\exp \left(t_{1} x_{1}\right) \ldots \exp \left(t_{n} x_{n}\right) s\right) \varphi_{0} .
$$

Proof. The map

$$
\left(t_{1}, \ldots, t_{n}, s\right) \mapsto \rho\left(\exp \left(t_{1} x_{1}\right) \ldots \exp \left(t_{n} x_{n}\right) s\right) \varphi_{0},
$$

defined for small enough $t_{j}$, is a smooth $\mathcal{H}_{K}$-valued map (cf. Remark A.9). Hence

$$
\left.\frac{\partial t^{n}}{\partial t_{n} \ldots \partial t_{1}}\right|_{t_{n}=\cdots=t_{1}=0} \rho\left(\exp \left(t_{n} x_{n}\right) \ldots \exp \left(t_{1} x_{1}\right) s\right) \varphi_{0} \in \mathcal{H}_{K}
$$

and

$$
\begin{aligned}
& \left(\left.\frac{\partial t^{n}}{\partial t_{n} \ldots \partial t_{1}}\right|_{t_{n}=\cdots=t_{1}=0} \rho\left(\exp \left(t_{n} x_{n}\right) \ldots \exp \left(t_{1} x_{1}\right) s\right) \varphi_{0}\right)\left(s_{0}\right) \\
& =\left.\frac{\partial t^{n}}{\partial t_{n} \ldots \partial t_{1}}\right|_{t_{n}=\cdots=t_{1}=0}\left(\rho(s) \varphi_{0}\right)\left(s_{0} \exp \left(t_{n} x_{n}\right) \ldots \exp \left(t_{1} x_{1}\right)\right) \\
& =\left(\mathcal{L}_{x_{n}} \ldots \mathcal{L}_{x_{1}}\left(\rho(s) \varphi_{0}\right)\right)\left(s_{0}\right) .
\end{aligned}
$$

Proposition 4.4. The domain $\mathcal{D}$ contains $\mathcal{H}_{K}^{0}$ and is dense in $\mathcal{H}_{K}$. The map $\alpha: \mathfrak{g}_{c} \rightarrow \operatorname{End}(\mathcal{D})$ is a strongly continuous representation of $\mathfrak{g}_{c}=\mathfrak{h} \oplus i \mathfrak{q}$ by skewsymmetric operators in the sense that all the maps $\mathfrak{g}_{c} \rightarrow \mathcal{H}_{K}, x \mapsto \alpha(x) v$ are continuous. More generally, for each $\varphi \in \mathcal{D}^{1}:=\bigcap_{x \in \mathfrak{g}} \mathcal{D}_{x}$, the map $\mathfrak{g}_{\mathbb{C}} \rightarrow \mathcal{H}_{K}, x \mapsto$ $\mathcal{L}_{x} \varphi$ is continuous.

Proof. The first assertion follows from (12) and Lemma 4.3. The map $\alpha$ is a Lie algebra homomorphism because $x \mapsto \mathcal{L}_{x}$ is so by (10). For the strong continuity it suffices to show that, for $\varphi \in \mathcal{D}^{1}$, the graph of the map $\mathfrak{g}_{c} \rightarrow \mathcal{H}_{K}, x \mapsto \mathcal{L}_{x} \varphi$ is closed (cf. [Ne10a, Lemma 4.2]). This follows if, for each $s \in S$ the map

$$
\mathfrak{g}_{c} \rightarrow \mathcal{H}, x \mapsto\left(\mathcal{L}_{x} \varphi\right)(s)=d \varphi(s) V_{x}(s)
$$

is continuous. That this is the case on the real Lie algebra $\mathfrak{g}$ follows from the fact that $\varphi$ is a smooth function on $S$ because $\mathfrak{g} \rightarrow T_{s}(S), x \mapsto V_{x}(s)$ is a topological isomorphism (cf. Definition A.8). Clearly, the continuity is inherited by the complex linear extension to $\mathfrak{g}_{\mathbb{C}}$. This completes the proof.

To show that the representation of $\mathfrak{g}_{c}$ integrates to a continuous unitary representation of $G_{c}$ we will use the following theorem

Theorem 4.5 ([Mer10]). Let $G_{c}$ be a simply connected Banach-Lie group with Lie algebra $\mathfrak{g}_{c}$. Assume that $\mathfrak{g}_{c}=\mathfrak{a}_{1} \oplus \mathfrak{a}_{2}$ where $\mathfrak{a}_{1}$ and $\mathfrak{a}_{2}$ are closed subspaces. Let $\alpha$ be a strongly continuous representation of $\mathfrak{g}_{c}$ on a dense subspace $\mathcal{D}$ of a Hilbert space $\mathcal{H}$ such that for every $x \in \mathfrak{a}_{1} \cup \mathfrak{a}_{2}, \alpha(x)$ is essentially skew-adjoint, $e^{\overline{\alpha(x)}} \mathcal{D} \subseteq \mathcal{D}$ and

$$
e^{\overline{\alpha(x)}} \alpha(y) e^{-\overline{\alpha(x)}}=\alpha\left(e^{\operatorname{ad} x} y\right) \quad \text { for } \quad y \in \mathfrak{g}_{c} .
$$

Then $\alpha$ integrates to a continuous unitary representation $(\pi, \mathcal{H})$ of $G_{c}$ for which $\mathcal{D} \subseteq$ $\mathcal{H}^{\infty}(\pi)$ is a $G_{c}$-invariant subspace and $\alpha(x)=\left.\mathrm{d} \pi(x)\right|_{\mathcal{D}}$ for $x \in \mathfrak{g}_{c}$. In particular, for $x \in \mathfrak{g}_{c}$, the infinitesimal generator $\overline{\mathrm{d} \pi}(x)$ of the one-parameter group $\pi(\exp t x)$ coincides with the closure $\overline{\alpha(x)}$. 
The next two propositions ensure that the assumptions of the theorem are satisfied by the representation $\alpha$ for $\mathfrak{a}_{1}=\mathfrak{h}$ and $\mathfrak{a}_{2}=i \mathfrak{q}$. The first one uses the Geometric Fröhlich Theorem 2.5.

Proposition 4.6. For $x \in \mathfrak{h} \cup i \mathfrak{q}$, the operator $\alpha(x)$ is essentially skew-adjoint with closure $\mathcal{L}_{x}^{K}$.

Proof. For $x \in \mathfrak{h} \cup i W$, we know that $\mathcal{H}_{K}^{0}$ is a core for the skew-adjoint operator $\overline{\mathrm{d} \rho}(x)=\mathcal{L}_{x}^{K}$ (Proposition 4.2). Since $\mathcal{H}_{K}^{0} \subseteq \mathcal{D} \subseteq \mathcal{D}_{x}$, the larger subspace $\mathcal{D}$ is also a core for $\mathcal{L}_{x}^{K}$. This proves the assertion for $x \in \mathfrak{h} \cup i W$.

Now let $x \in \mathfrak{q}$ be a general element. Then the vector field $V_{x}$ is $K$-symmetric, since for every $s_{1}, s_{2} \in S$, we have

$$
K\left(s_{1} \exp t x, s_{2}\right)=\varphi_{0}\left(s_{1}(\exp t x) s_{2}^{*}\right)=\varphi_{0}\left(s_{1}\left(s_{2} \exp t x\right)^{*}\right)=K\left(s_{1}, s_{2} \exp t x\right) .
$$

We can therefore apply Theorem 2.5 and Proposition 4.2 to see that $\mathcal{L}_{x}^{K}$ is a selfadjoint operator, and that $\left.\mathcal{L}_{x}\right|_{\mathcal{H}_{K}^{0}}$ is essentially self-adjoint. Writing $x=x_{+}-x_{-}$ with $x_{ \pm} \in W$, we see that we also have $\mathcal{L}_{x}=\mathcal{L}_{x_{+}}-\mathcal{L}_{x_{-}}$, so that

$$
\mathcal{H}_{K}^{0} \subseteq \mathcal{D} \subseteq \mathcal{D}_{x_{+}} \cap \mathcal{D}_{x_{-}} \subseteq \mathcal{D}_{x}
$$

and thus $\alpha(i x)$ is essentially skew-adjoint with closure $\mathcal{L}_{i x}^{K}$.

Lemma 4.7. Let $x \in \mathfrak{h} \cup W, y \in \mathfrak{g}$, and $\varphi \in \mathcal{H}_{K}$ such that $\varphi \in \mathcal{D}_{e^{-\operatorname{ad} x} y}$. Then $\rho(\exp x) \varphi \in \mathcal{D}_{y}$ and

$$
\mathcal{L}_{y}(\rho(\exp x) \varphi)=\rho(\exp x) \mathcal{L}_{e^{-\operatorname{ad} x_{y}} \varphi}
$$

Proof. For every $s \in S, \mathcal{L}_{e^{- \text {ad } x} y} \varphi \in \mathcal{H}$ leads to

$$
\begin{aligned}
& \left\langle\rho(\exp x) \mathcal{L}_{e^{-\operatorname{ad} x} y} \varphi, K_{s}\right\rangle \\
= & \left\langle\mathcal{L}_{e^{-\operatorname{ad} x} y} \varphi, \rho\left((\exp x)^{*}\right) K_{s}\right\rangle=\left\langle\mathcal{L}_{e^{-\operatorname{ad} x} y} \varphi, K_{s \exp x}\right\rangle \\
= & \left.\frac{d}{d t}\right|_{t=0} \varphi\left(s \exp x \exp \left(t e^{-\operatorname{ad} x} y\right)\right)=\left.\frac{d}{d t}\right|_{t=0} \varphi(s \exp t y \exp x) \\
= & \mathcal{L}_{y}(\rho(\exp x) \varphi)(s)=\left\langle\mathcal{L}_{y}(\rho(\exp x) \varphi), K_{s}\right\rangle .
\end{aligned}
$$

Lemma 4.8. Let $A: \mathcal{D} \rightarrow \mathcal{H}$ be a symmetric operator and $\mathcal{D}_{1} \subseteq \mathcal{D}$ a dense subspace. If $A_{1}:=\left.A\right|_{\mathcal{D}_{1}}$ is essentially self-adjoint, then $A$ is also essentially selfadjoint, with $\bar{A}=\overline{A_{1}}$.

Proof. From $A_{1} \subseteq A \subseteq A^{*}$ it follows that

$$
\bar{A} \subseteq A^{*} \subseteq A_{1}^{*}=\overline{A_{1}} \subseteq \bar{A}
$$

(cf. [RS80, Thm. VIII.1]). Therefore $\bar{A}=A^{*}=\overline{A_{1}}$ is self-adjoint.

Proposition 4.9. For $x \in \mathfrak{h} \cup i \mathfrak{q}, e^{\overline{\alpha(x)}} \mathcal{D} \subseteq \mathcal{D}$. Moreover for any $y \in \mathfrak{g}_{c}$ we have

$$
e^{\overline{\alpha(x)}} \alpha(y) e^{-\overline{\alpha(x)}}=\alpha\left(e^{\operatorname{ad} x} y\right) \text {. }
$$

Proof. In the following we also write $\rho(h):=\rho_{H}(h)$ for $h \in H$.

Step 1: Let $x \in \mathfrak{h} \cup W$ and $\varphi \in \mathcal{D}$. Then Lemma 4.7 implies by induction that for every $n \in \mathbb{N}$ and $y_{1}, \ldots, y_{n} \in \mathfrak{h} \cup W$,

$$
\mathcal{L}_{y_{n}} \ldots \mathcal{L}_{y_{1}} \rho(\exp x) \varphi=\rho(\exp x) \mathcal{L}_{e^{-\operatorname{ad} x} y_{n}} \ldots \mathcal{L}_{e^{-\operatorname{ad} x} y_{1}} \varphi \in \mathcal{H},
$$

and hence $e^{\overline{\mathrm{d} \rho}(x)} \varphi=\rho(\exp x) \varphi \in \mathcal{D}\left(\overline{\mathrm{d} \rho}\left(y_{n}\right) \ldots \overline{\mathrm{d} \rho}\left(y_{1}\right)\right)$. It follows that

$$
e^{\overline{\mathrm{d} \rho}(x)} \mathcal{D} \subseteq \mathcal{D},
$$

and that we have for $\varphi \in \mathcal{D}$ and (by linearity) for $y \in \mathfrak{g}_{c}$,

$$
\alpha(y) e^{\overline{\mathrm{d} \rho}(x)} \varphi=e^{\overline{\mathrm{d} \rho}(x)} \alpha\left(e^{-\mathrm{ad} x} y\right) \varphi .
$$


In particular (13) holds for $x \in \mathfrak{h}$. Now let $\psi \in \mathcal{D}$. Then (14) can be written

$$
\left\langle-e^{\overline{\mathrm{d} \rho}(x)} \varphi, \alpha(y) \psi\right\rangle=\left\langle e^{\overline{\mathrm{d} \rho}(x)} \alpha\left(e^{-\mathrm{ad} x} y\right) \varphi, \psi\right\rangle,
$$

and this last equation is all we need for the following.

Step 2: Assume that $x \in W$. Then the spectrum of $\overline{\mathrm{d} \rho}(x)$ is bounded from above and hence $t \mapsto e^{t \overline{\mathrm{d} \rho}(x)}$ extends to a strongly continuous holomorphic semigroup

$$
\mathbb{C}^{+}=\{z \in \mathbb{C} \mid \operatorname{Re} z \geq 0\} \rightarrow B(\mathcal{H}), z \mapsto e^{z \overline{\mathrm{d} \rho}(x)},
$$

which is holomorphic on int $\left(\mathbb{C}^{+}\right)$(cf. [HN93, Prop. 9.9] or [Ne00, Prop. VI.3.2]). For $\varphi \in \mathcal{D}^{1}$, the map $\mathfrak{g}_{\mathbb{C}} \rightarrow \mathcal{H}, x \mapsto \mathcal{L}_{x} \varphi$ is $\mathbb{C}$-linear and continuous (Proposition 4.4), hence the function $\mathbb{C} \rightarrow \mathcal{H}, z \mapsto \mathcal{L}_{e^{-z \text { ad } x} y} \varphi$ is analytic. Since the map

$$
B(\mathcal{H}) \times \mathcal{H} \rightarrow \mathcal{H}, \quad(T, \xi) \mapsto T \xi
$$

is $\mathbb{C}$-bilinear and continuous, it follows that the map

$$
\operatorname{int}\left(\mathbb{C}^{+}\right) \rightarrow \mathcal{H}, \quad z \mapsto e^{z \overline{\mathrm{d} \rho}(x)} \mathcal{L}_{e^{-z \text { ad } x} y} \varphi
$$

is analytic. By the Analytic Continuation Principle, the equality (15) implies

$$
\left\langle-e^{z \overline{\mathrm{d} \rho}(x)} \varphi, \alpha(y) \psi\right\rangle=\left\langle e^{z \overline{\mathrm{d} \rho}(x)} \mathcal{L}_{e^{-z \operatorname{ad} x} y} \varphi, \psi\right\rangle \quad \text { for } z \in \operatorname{int} \mathbb{C}^{+}, \psi \in \mathcal{D} .
$$

We then have by continuity

$$
\left\langle-e^{ \pm i \overline{\mathrm{d} \rho}(x)} \varphi, \alpha(y) \psi\right\rangle=\left\langle e^{ \pm i \overline{\mathrm{d} \rho}(x)} \mathcal{L}_{e^{\mp i \text { ad } x} y} \varphi, \psi\right\rangle .
$$

This shows that, for $y \in \mathfrak{h} \cup i W, e^{ \pm i \overline{\mathrm{d} \rho}(x)} \varphi \in \mathcal{D}\left(\alpha(y)^{*}\right)=\mathcal{D}(\overline{\mathrm{d} \rho}(y))=\mathcal{D}_{y}$. We thus arrive at

$$
e^{ \pm i \overline{\mathrm{d} \rho}(x)} \mathcal{D}^{1} \subseteq \mathcal{D}^{1} \quad \text { with } \quad \mathcal{L}_{y} e^{ \pm i \overline{\mathrm{d} \rho}(x)}=e^{ \pm i \overline{\mathrm{d} \rho}(x)} \mathcal{L}_{e^{\mp i \text { ad } x} y} \quad \text { on } \quad \mathcal{D}^{1} .
$$

By induction, we now obtain

$$
e^{ \pm i \overline{\mathrm{d} \rho}(x)} \mathcal{D}=\mathcal{D} \quad \text { with }\left.\quad \alpha(y) e^{ \pm i \overline{\mathrm{d} \rho}(x)}\right|_{\mathcal{D}}=e^{ \pm i \overline{\mathrm{d} \rho}(x)} \alpha\left(e^{\mp i \mathrm{ad} x} y\right)
$$

Step 3: For $n \in \mathbb{N}, x_{1}, x_{2} \in W, y \in \mathfrak{g}_{c}$ and $\varphi, \psi \in \mathcal{D}^{1}$ we now obtain

$$
\begin{aligned}
& \left\langle-\left(e^{i \overline{\mathrm{d} \rho}\left(x_{1}\right)} e^{-i \overline{\mathrm{d} \rho}\left(x_{2}\right)}\right)^{n} \varphi, \overline{\mathrm{d} \rho}(y) \psi\right\rangle \\
& \quad=\left\langle\mathcal{L}_{\left(e^{i \text { ad } x_{2}} e^{-i \operatorname{ad} x_{1}}\right)^{n}{ }_{y} \varphi},\left(e^{i \overline{\mathrm{d} \rho}\left(x_{2}\right)} e^{-i \overline{\mathrm{d} \rho}\left(x_{1}\right)}\right)^{n} \psi\right\rangle
\end{aligned}
$$

from

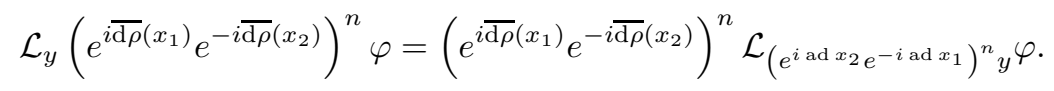

Now let $x \in \mathfrak{q}$ and write it as $x=x_{1}-x_{2}$ with $x_{1}, x_{2} \in W$. Since $\overline{\mathrm{d} \rho}\left(x_{1}\right)-\overline{\mathrm{d} \rho}\left(x_{2}\right)$ is essentially self-adjoint on $\mathcal{D}$ (Proposition 4.6), it is essentially self-adjoint as an operator on its domain $\mathcal{D}\left(\overline{\mathrm{d} \rho}\left(x_{1}\right)\right) \cap \mathcal{D}\left(\overline{\mathrm{d} \rho}\left(-x_{2}\right)\right)$, and its closure is $\overline{\alpha\left(x_{1}-x_{2}\right)}$ (Lemma 4.8). We therefore have Trotter's Product Formula [RS80, Thm. VIII.31]:

$$
\lim _{n \rightarrow \infty}\left(e^{i \frac{\overline{\mathrm{d} \rho}\left(x_{1}\right)}{n}} e^{-i \frac{\overline{\mathrm{d} \rho}\left(x_{2}\right)}{n}}\right)^{n} f=e^{i \overline{\alpha\left(x_{1}-x_{2}\right)}} f \quad \text { for } \quad f \in \mathcal{H}_{K} .
$$

Replacing $x_{j}$ by $\frac{x_{j}}{n}, j=1,2$, in (17) and taking the limit we obtain

$$
\left\langle-e^{\overline{i \alpha\left(x_{1}-x_{2}\right)}} \varphi, \mathcal{L}_{y} \psi\right\rangle=\left\langle e^{\overline{i \alpha\left(x_{1}-x_{2}\right)}} \mathcal{L}_{e^{i \operatorname{ad}\left(x_{2}-x_{1}\right)} y} \varphi, \psi\right\rangle \quad \text { for } \quad \varphi, \psi \in \mathcal{D}^{1}
$$

As above we now obtain by induction that $e^{i \overline{\alpha(x)}} \mathcal{D} \subseteq \mathcal{D}$ and that (13) holds for $x \in i \mathfrak{q}$. 
Proof of Theorem 3.7: In view of Proposition 4.9, we obtain from Theorem 4.5 a unitary representation of $G_{c}$ whose space of smooth vectors contains $\mathcal{D}$ and such that $\left.\mathrm{d} \pi\right|_{\mathcal{D}}=\alpha$ and $\overline{\mathrm{d} \pi}(x)=\overline{\alpha(x)}$ for $x \in \mathfrak{g}_{c}$. We conclude with Proposition 4.2 and 4.6 that $\overline{\mathrm{d} \pi}(x)=\overline{\mathrm{d} \rho}(x)$ for $x \in \mathfrak{h}$ and $\overline{\mathrm{d} \pi}(i x)=i \overline{\mathrm{d} \rho}(x)$ for $x \in W$.

Remark 4.10. Since $\mathcal{D} \subseteq \mathcal{H}^{\infty}(\pi)$, it immediatly follows from

$$
\mathcal{H}^{\infty}(\pi)=\bigcap_{x_{j} \in \mathfrak{g}_{c}, \bigcap_{n \in \mathbb{N}}} \mathcal{D}\left(\overline{\mathrm{d} \pi}\left(x_{n}\right) \ldots \overline{\mathrm{d} \pi}\left(x_{1}\right)\right) \subseteq \mathcal{D}
$$

([Ne10b, Lemma 3.4, Remark 8.3]), that $\mathcal{D}=\mathcal{H}^{\infty}(\pi)$.

\section{HOLOMORPHIC EXTENSION OF SEMIBOUNDED REPRESENTATIONS}

In this section we obtain a result which is a converse to the Lüscher-Mack Theorem. It is new even in the finite dimensional setting. At the same time, we obtain the existence of holomorphic extensions for semibounded unitary representations.

Definition 5.1. Let $G$ be a Banach-Lie group with Lie algebra $\mathfrak{g}$. For a smooth unitary representation $(\pi, \mathcal{H})$ of $G$ we consider the map

$$
s_{\pi}: \mathfrak{g} \rightarrow \mathbb{R} \cup\{\infty\}, \quad s_{\pi}(x):=\sup (\operatorname{Spec}(i \overline{\mathrm{d} \pi}(x))) .
$$

(a) A smooth unitary representation $(\pi, \mathcal{H})$ of $G$ is called semibounded if $s_{\pi}$ is bounded on a non-empty open subset of $\mathfrak{g}$. Then the cone $W_{\pi}$, consisting of all those $x \in \mathfrak{g}$ for which $s_{\pi}$ is bounded on a neighborhood of $x$, is an open $\operatorname{Ad}(G)$ invariant convex cone in $\mathfrak{g}$. Moreover, $s_{\pi}: W_{\pi} \rightarrow \mathbb{R}$ is a continuous convex function $([\mathrm{Ne} 08])$.

For a convex cone $W \subseteq \mathfrak{g}$, we say that $\pi$ is $W$-semibounded if $s_{\pi}(W) \subseteq \mathbb{R}$ and $s_{\pi}: W \rightarrow \mathbb{R}$ is locally bounded.

(b) A convex cone $W \subseteq \mathfrak{g}$ is said to be relatively open if the linear subspace $W-W$ of $\mathfrak{g}$ is closed and $W$ is open in $W-W$.

For the definition of a $C^{1}$-map we use in the next lemma see Definition B.2.

Lemma 5.2. Let $W$ be a relatively open convex cone in $\mathfrak{g}, \mathfrak{q}:=W-W$, and $(\pi, \mathcal{H})$ be a smooth $W$-semibounded unitary representation of $G$. Then, for every $v \in \mathcal{H}^{\infty}(\pi)$, the map

is $C^{1}$ and

$$
\rho^{v}: W \rightarrow \mathcal{H}, x \mapsto e^{i \overline{\mathrm{d} \pi}(x)} v,
$$

$$
T_{x}\left(\rho^{v}\right)(y)=\overline{\mathrm{d} \pi}\left(\int_{0}^{1} e^{s \mathrm{ad} i x} y d s\right) e^{i \overline{\mathrm{d} \pi}(x)} v .
$$

The map $G \times W \rightarrow \mathcal{H},(g, x) \mapsto \pi(g) \rho^{v}(x)$ is also $C^{1}$.

Proof. For $x \in W, y \in \mathfrak{g}_{c}$ and $v, w \in \mathcal{H}^{\infty}$, the function

$$
F(z):=\left\langle-e^{z \overline{\mathrm{d} \pi}(x)} v, \mathrm{~d} \pi(y) w\right\rangle-\left\langle e^{z \overline{\mathrm{d} \pi}(x)} \mathrm{d} \pi\left(e^{-z \mathrm{ad} x} y\right) v, w\right\rangle
$$

is continuous on the closed upper half-plane $\mathbb{C}_{+}:=\{z \in \mathbb{C}: \operatorname{Im} z \geq 0\}$ and holomorphic on its interior ([Ne00, Prop. VI.3.2]; see also Step 2 in the proof of Proposition 4.9). Since $F(t)=0$ for $t \in \mathbb{R}$ by Proposition 4.9, the Schwarz Reflection Principle [Ru87, Thm. 11.14] implies that $F$ vanishes on $\mathbb{C}_{+}$. It follows that, for $x \in W$ and $v \in \mathcal{H}^{\infty}$ we have $e^{i \overline{\mathrm{d} \pi(x)}} v \in \mathcal{D}(\overline{\mathrm{d} \pi(y)})$ for every $y \in \mathfrak{g}$ with

$$
\overline{\mathrm{d} \pi}(y) e^{i \overline{\mathrm{d} \pi}(x)} v=e^{i \overline{\mathrm{d} \pi}(x)} \mathrm{d} \pi\left(e^{-\mathrm{ad} i x} y\right) v .
$$

Since $\mathrm{d} \pi\left(e^{-\operatorname{ad} i x} y\right) v$ is again a smooth vector, we can iterate this argument to obtain inductively

$$
e^{i \overline{\mathrm{d} \pi}(x)} v \in \mathcal{D}:=\bigcap_{y_{n}, \ldots, y_{1} \in \mathfrak{g}, n \in \mathbb{N}} \mathcal{D}\left(\overline{\mathrm{d} \pi}\left(y_{n}\right)\right) \ldots \mathcal{D}\left(\overline{\mathrm{d} \pi}\left(y_{1}\right)\right)
$$


We know by [Ne10b, Lemma 3.4, Remark 8.3] that $\mathcal{D}=\mathcal{H}^{\infty}$. Hence we have

$$
e^{i \overline{\mathrm{d} \pi}(x)} \mathcal{H}^{\infty} \subseteq \mathcal{H}^{\infty} \text { and } \mathrm{d} \pi(y) e^{i \overline{\mathrm{d} \pi}(x)}=e^{i \overline{\mathrm{d} \pi}(x)} \mathrm{d} \pi\left(e^{-\operatorname{ad} i x} y\right)
$$

for $x \in W$ and $y \in \mathfrak{g}_{\mathbb{C}}$. Now let $v \in \mathcal{H}^{\infty}$. In view of (20), Proposition B.5 implies that $\rho^{v}$ is $C^{1}$ with

$$
T_{x}\left(\rho^{v}\right)(y)=\mathrm{d} \pi\left(\int_{0}^{1} e^{s \operatorname{ad} i x} y d s\right) e^{i \overline{\mathrm{d} \pi}(x)} v=e^{i \overline{\mathrm{d} \pi}(x)} \mathrm{d} \pi\left(\int_{0}^{1} e^{-s \operatorname{ad} i x} y d s\right) v
$$

and that the map

is continuous.

$$
\widehat{\rho}: W \times \mathcal{H} \rightarrow \mathcal{H}, \quad(x, v) \mapsto e^{i \overline{\mathrm{d} \pi}(x)} v
$$

Finally, we observe that the map

$$
F: G \times W \rightarrow \mathcal{H}, \quad F(g, x):=\pi(g) \rho^{v}(x)
$$

is continuous because the $G$-action on $\mathcal{H}$ defined by $\pi$ is continuous. We have just seen that $F$ is partially differentiable in $x$ with continuous partial derivative

$$
G \times W \times \mathfrak{q} \rightarrow \mathcal{H}, \quad(g, x, y) \mapsto \pi(g) T_{x}\left(\rho^{v}\right) y .
$$

We have also seen that $\rho^{v}(W) \subseteq \mathcal{H}^{\infty}(\pi)$, so that the partial derivatives in $g$ also exist and are given by

$$
T G \times W \rightarrow \mathcal{H}, \quad(g . y, x) \mapsto \pi(g) \mathrm{d} \pi(y) e^{i \overline{\mathrm{d} \pi}(x)} v=\pi(g) e^{i \overline{\mathrm{d} \pi}(x)} \mathrm{d} \pi\left(e^{-\operatorname{ad}(i x)} y\right) v .
$$

As $G$ acts continously on $\mathcal{H}, \widehat{\rho}$ is continuous, and the adjoint action of $G$ on $\mathfrak{g}$ is continuous, this function is continuous. This implies that $F$ is $C^{1}$ (cf. [Ham82]).

Definition 5.3. Let $W$ be a relatively open convex cone in $\mathfrak{g}, \mathfrak{q}:=W-W$, and $\mathfrak{h}$ be a closed subalgebra of $\mathfrak{g}$. The cone $W$ is called $\mathfrak{h}$-compatible if

$$
[W, W] \subseteq \mathfrak{h} \quad \text { and } \quad e^{\text {ad } \mathfrak{h}} W \subseteq W .
$$

Then $\mathfrak{g}_{c}:=\mathfrak{h} \oplus i \mathfrak{q} \subseteq \mathfrak{g}_{\mathbb{C}}$ is a closed subalgebra which is turned in a symmetric Banach-Lie algebra by the involution $\theta(x+i y):=x-i y$ for $x \in \mathfrak{h}, y \in \mathfrak{q}$.

An $\mathfrak{h}$-compatible cone $W \subseteq \mathfrak{g}$ is said to be integrable if there exists a symmetric Banach-Lie group $\left(G_{c}, \theta\right)$ with symmetric Lie algebra $\mathfrak{g}_{c}=\mathfrak{h} \oplus i \mathfrak{q}$ such that, for $H:=\left(G_{c}^{\theta}\right)_{0}$, the polar map

$$
H \times i W \rightarrow G_{c}, \quad(h, x) \mapsto h \exp (x)
$$

is an analytic diffeomorphism onto an open subsemigroup

$$
S=S_{H}(i W)=H \exp (i W) \subseteq G .
$$

Then $S$ is invariant under the involution $s^{*}=\theta(s)^{-1}$, turning it into an involutive semigroup $(S, *)$.

From the discussion of Banach-Olshanski semigroups in Appendix A, it then follows that for each connected Banach-Lie group $H_{1}$ locally isomorphic to $H$ to which the adjoint action of $\mathfrak{h}$ on $\mathfrak{g}_{c}$ integrates, there exists an involutive BanachOlshanski semigroup $S_{H_{1}}(i W)$ with a polar decomposition which is a quotient of the universal covering semigroup of $S_{H}(i W)$.

Theorem 5.4. Let $G$ be a Banach-Lie group with Lie algebra $\mathfrak{g}, \mathfrak{h}$ be a closed complemented Lie subalgebra of $\mathfrak{g}$, and $H:=\langle\exp \mathfrak{h}\rangle$ be the corresponding integral subgroup in $G$. Let $(\pi, \mathcal{H})$ be a smooth $W$-semibounded unitary representation of $G$ for the integrable $\mathfrak{h}$-compatible cone $W$. Then the formula

$$
\rho(h \exp i x):=\pi(h) e^{i \overline{\mathrm{d} \pi}(x)} \text { for } h \in H \text { and } x \in W,
$$

defines a strongly continuous smooth $*$-representation $\rho$ of $S_{H}(i W)$ on $\mathcal{H}$.

We shall need the following Chain Rule ([Mer10, Lemma 4]): 
Lemma 5.5. Let $I \subseteq \mathbb{R}$ be an open interval, $E$ and $F$ be two Banach spaces and $L_{s}(E, F)$ denotes the space of continuous linear operators from $E$ to $F$ endowed with the strong operator topology. Let $I \rightarrow L_{s}(E, F), t \mapsto K(t)$ be a continuous path such that $t \mapsto K(t) v$ is differentiable for every $v$ in a subspace $\mathcal{D}$ of $E$ and let $\gamma(t)$ be a differentiable path in $\mathcal{D}$. We write $K^{\prime}(t): \mathcal{D} \rightarrow F$ for the linear operator obtained by $K^{\prime}(t) v:=\frac{d}{d t} K(t) v$ for $v \in \mathcal{D}$. Then $t \mapsto K(t) \gamma(t)$ is differentiable with

$$
\frac{d}{d t} K(t) \gamma(t)=K^{\prime}(t) \gamma(t)+K(t) \gamma^{\prime}(t)
$$

Proof of Theorem 5.4. Step 1: Let us first prove that, for $x_{1}, x_{2} \in W$,

$$
\rho\left(\exp i x_{1} \exp i x_{2}\right)=\rho\left(\exp i x_{1}\right) \rho\left(\exp i x_{2}\right) .
$$

For this purpose, let us write for $t>0$,

$$
\eta(t):=\exp t i x_{1} \exp i x_{2}=h_{t} \exp (i x(t)) \text { with } h_{t} \in H, x(t) \in W .
$$

Now let $v \in \mathcal{H}^{\infty}$ and consider $\gamma(t):=\rho(\eta(t)) v=\pi\left(h_{t}\right) e^{i \overline{\mathrm{d} \pi}(x(t))} v$. By Lemma 5.2 and the Chain Rule (Lemma 5.5), applied with $K(t)=\pi\left(h_{t}\right)$ and $\mathcal{D}=\mathcal{H}^{\infty}(\pi)$, the path $\gamma(t)$ is differentiable for $t>0$, and, denoting by $\delta$ the right logarithmic derivative (see Definition A.11), we obtain with Proposition A.12

$$
\begin{aligned}
\gamma^{\prime}(t) & =\overline{\mathrm{d} \pi}\left(\delta h_{t}\right) \gamma(t)+\pi\left(h_{t}\right) \overline{\mathrm{d} \pi}\left(\int_{0}^{1} e^{i s \operatorname{ad} x(t)} i x^{\prime}(t) d s\right) e^{\overline{\mathrm{d} \pi}(i x(t))} v \\
& =\overline{\mathrm{d} \pi}\left(\delta h_{t}\right) \gamma(t)+\pi\left(h_{t}\right) \overline{\mathrm{d} \pi}\left(\delta(\exp )_{i x(t)} i x^{\prime}(t)\right) e^{\overline{\mathrm{d} \pi}(i x(t))} v \\
& =\overline{\mathrm{d} \pi}\left(\delta(h)_{t}+\operatorname{Ad}\left(h_{t}\right) \delta(\exp )_{i x(t)} i x^{\prime}(t)\right) \gamma(t) \\
& =\overline{\mathrm{d} \pi}\left(\delta(\eta)_{t}\right) \gamma(t) \quad(\text { by Proposition A.13) } \\
& =\overline{\mathrm{d} \pi}\left(i x_{1}\right) \gamma(t) .
\end{aligned}
$$

Since $\lim _{t \rightarrow 0} \gamma(t)=\lim _{t \rightarrow 0} \pi\left(h_{t}\right) e^{i \overline{\mathrm{d} \pi}(x(t))} v=\rho\left(\exp i x_{2}\right) v($ Lemma 5.2, Lemma A.6), we obtain with [Kat66, p. 481] that

$$
\gamma(t)=\rho\left(\exp t i x_{1}\right) \rho\left(\exp i x_{2}\right) v,
$$

and (21) follows for $t=1$.

Step 2: For $h \in H$ and $x \in W$ we have

$$
\pi(h) \overline{\mathrm{d} \pi}(x) \pi(h)^{-1}=\overline{\mathrm{d} \pi}(\operatorname{Ad}(h) x),
$$

so that

$$
e^{\overline{\mathrm{d} \pi}(i \operatorname{Ad}(h) x)}=e^{\pi(h) i \overline{\mathrm{d} \pi}(x) \pi(h)^{-1}}=\pi(h) e^{i \overline{\mathrm{d} \pi}(x)} \pi(h)^{-1}
$$

From (23) we obtain the relation

$$
\rho(h \exp x)^{*}=\rho\left((h \exp x)^{*}\right),
$$

and we further derive

$$
\rho(s h)=\rho(s) \pi(h) \quad \text { for } \quad s \in S, h \in H .
$$

Step 3: Now we can prove that for $h_{1}, h_{2} \in H$ and $x_{1}, x_{2} \in W$,

$$
\rho\left(h_{1} \exp i x_{1} h_{2} \exp i x_{2}\right)=\rho\left(h_{1} \exp i x_{1}\right) \rho\left(h_{2} \exp i x_{2}\right) .
$$


With (21) and (23) we obtain

$$
\begin{aligned}
\rho\left(h_{1} \exp i x_{1}\right) \rho\left(h_{2} \exp i x_{2}\right) & =\pi\left(h_{1}\right) e^{\overline{\mathrm{d} \pi}\left(i x_{1}\right)} \pi\left(h_{2}\right) e^{i \overline{\mathrm{d} \pi}\left(x_{2}\right)} \\
& =\pi\left(h_{1}\right) \pi\left(h_{2}\right) e^{i \overline{\mathrm{d} \pi}\left(\operatorname{Ad}\left(h_{2}\right)^{-1} x_{1}\right)} e^{i \overline{\mathrm{d} \pi}\left(x_{2}\right)} \\
& =\pi\left(h_{1} h_{2}\right) \rho\left(\exp \left(i \operatorname{Ad}\left(h_{2}\right)^{-1} x_{1}\right)\right) \rho\left(\exp \left(i x_{2}\right)\right) \\
& =\pi\left(h_{1} h_{2}\right) \rho\left(\exp \left(i \operatorname{Ad}\left(h_{2}\right)^{-1} x_{1}\right) \exp \left(i x_{2}\right)\right) \\
& =\rho\left(h_{1} h_{2} \exp \left(i \operatorname{Ad}\left(h_{2}\right)^{-1} x_{1}\right) \exp \left(i x_{2}\right)\right) \\
& \left.=\rho\left(h_{1} \exp i x_{1}\right) h_{2} \exp i x_{2}\right) .
\end{aligned}
$$

Step 4: It remains to prove that for $v \in \mathcal{H}^{\infty}(\pi)$ the map $\rho^{v}: S \rightarrow \mathcal{H}, \rho^{v}(s):=$ $\rho(s) v$ is smooth. In view of Lemma 5.2, this map is $C^{1}$. For $x \in \mathfrak{h}$ we have by Remark A.9 and (24)

$$
T_{s}\left(\rho^{v}\right)(s . x):=\left.\frac{d}{d t}\right|_{t=0} \rho(s \exp t x) v=\left.\frac{d}{d t}\right|_{t=0} \rho(s) \rho(\exp t x) v=\rho(s) \mathrm{d} \pi(x) v .
$$

Similarly we have for $x \in W$

$$
T_{s}\left(\rho^{v}\right)(s .(i x)):=\left.\frac{d}{d t}\right|_{t=0} \rho(s \exp t i x) v=\left.\frac{d}{d t}\right|_{t=0} \rho(s) \rho(\exp t i x) v=\rho(s) \mathrm{d} \pi(i x) v .
$$

Since $T_{s}\left(\rho^{v}\right)$ linear, it follows that

$$
T_{s}\left(\rho^{v}\right)(s . x)=\rho(s) \mathrm{d} \pi(x) v \quad \text { for } x \in \mathfrak{h}+i \mathfrak{q} .
$$

Now an easy induction shows that the higher partial derivatives of $T \rho^{v}$ only involve the continuous $n$-linear maps

$$
\omega_{v}^{n}\left(x_{1}, \ldots, x_{n}\right):=\mathrm{d} \pi\left(x_{1}\right) \ldots \mathrm{d} \pi\left(x_{n}\right) v,
$$

and hence that $\rho^{v}$ is smooth.

Now recall the context of the Lüscher-Mack Theorem. We have a symmetric Banach-Lie algebra $\mathfrak{g}=\mathfrak{h} \oplus \mathfrak{q}$ and an integrable $e^{\text {ad } \mathfrak{h}}$-invariant open convex cone $W \subseteq \mathfrak{q}$. We therefore have a Banach-Olshanski semigroup $S_{H}(W)=H \exp W$ for each connected Lie group with Lie algebra $\mathfrak{h}$. Applying the preceding theorem to $\mathfrak{g}_{c}=\mathfrak{h}+i \mathfrak{q}$ and $-i W \subseteq i \mathfrak{q}$, we obtain the following converse to the Lüscher-Mack Theorem:

Corollary 5.6. Let $\mathfrak{g}=\mathfrak{h} \oplus \mathfrak{q}$ be a symmetric Banach-Lie algebra and $W$ be an integrable $e^{\text {ad } \mathfrak{h}}$-invariant open convex cone in $\mathfrak{q}$. Let $G_{c}$ be a Banach-Lie group with Lie algebra $\mathfrak{g}_{c}=\mathfrak{h}+i \mathfrak{q} \subseteq \mathfrak{g}_{\mathbb{C}}$ and let $H_{c}$ be its integral subgroup with Lie algebra $\mathfrak{h}$. Let $\pi$ be an-iW-semibounded unitary representation of $G_{c}$. Then

$$
\rho(h \exp x):=\pi(h) e^{\overline{\mathrm{d} \pi}(x)} \text { for } h \in H_{c} \text { and } x \in W,
$$

defines a strongly continuous smooth $*$-representation $\rho$ of $S_{H_{c}}(W)$.

Theorem 5.7 (Holomorphic Extension Theorem). Let $G$ be a Banach-Lie group with Lie algebra $\mathfrak{g},(\pi, \mathcal{H})$ be a semibounded unitary representation of $G$, and $W \subseteq$ $W_{\pi}$ be an open integrable $\operatorname{Ad}(G)$-invariant convex cone. Then

$$
\rho(g \exp i x):=\pi(g) e^{i \overline{\mathrm{d} \pi}(x)} \text { for } g \in G \text { and } x \in W,
$$

defines a holomorphic *-representation $\rho$ of the complex involutive semigroup

$$
S_{G}(i W)=G \exp i W .
$$

In particular the vectors in $\rho\left(S_{G}(i W)\right) \mathcal{H}$ are analytic for $\pi$.

Note that $S_{G}(i W)$ is a complex Olshanski semigroup (cf. Definition A.4). 
Proof. First we observe that the cone $W$ is $\mathfrak{g}$-compatible. Theorem 5.4 now applies to the semigroup $S_{G}(i W)$. It remains to prove that $\rho: S_{G}(i W) \rightarrow B(\mathcal{H})$ is holomorphic. But (26) shows that $T \rho^{v}$ is complex linear, hence that $\rho^{v}$ is holomorphic. The holomorphy of $\rho$ now follows from [Ne00, Lemma IV.2.2]. Now let $s \in S_{G}(i W)$ and $v \in \mathcal{H}$. Since $\pi^{\rho(s) v}(h)=\pi(h) \rho(s) v=\rho(h s) v$, the analyticity of $\pi^{\rho(s) v}$ follows from the analyticity of the map $H \rightarrow S, h \mapsto h s$.

The preceding theorem generalizes Olshanski's Holomorphic Extension Theorem for highest weight representations ([Ol82], [Ne00]) to the Banach-Lie setting. In the finite dimensional case the proof heavily relies on the existence of a dense space of analytic vectors, which can be derived by convolution with heat kernels ([Ga60]), but for unitary representations of Banach-Lie groups, not even the space of $C^{1}$ vectors need to be dense (cf. [Ne10b]). In the finite dimensional context one proves first that $\rho$ is holomorphic, and then the multiplicativity of $\rho$ is obtained by analytic continuation. In the proof we give here the multiplicativity of $\rho$ follows from the (assumed) existence of smooth vectors and then the holomorphy of $\rho$ follows as a bonus from its multiplicativity.

\section{Appendix A. Covering theory for Olshanski semigroups}

Let $(G, \theta)$ be a symmetric Banach-Lie group and $(\mathfrak{g}, \theta)$ the corresponding symmetric Lie algebra. We write

$$
\mathfrak{g}=\mathfrak{h} \oplus \mathfrak{q} \quad \text { with } \quad \mathfrak{h}=\operatorname{ker}(\theta-\mathbf{1}) \quad \text { and } \quad \mathfrak{q}=\operatorname{ker}(\theta+\mathbf{1}),
$$

for the eigenspace decomposition of $\mathfrak{g}$ under $\theta$ and let $H_{G}$ denote the identity component of $G^{\theta}$. We set $g^{*}=\theta(g)^{-1}$ and consider an open convex $\operatorname{Ad}\left(H_{G}\right)$ invariant convex cone $W \subseteq \mathfrak{q}$ for which the map

$$
H_{G} \times W \rightarrow G, \quad(h, x) \mapsto h \exp x
$$

is an analytic diffeomorphism onto an open subsemigroup $S=H \exp (W)$ of $G$. In these coordinates the involution on $h \exp x \in S$ is given by

$$
(h \exp x)^{*}=(\exp x) h^{-1}=h^{-1} \exp (\operatorname{Ad}(h) x) .
$$

In particular $S$ is $*$-invariant. In the following we write $S_{H_{G}}(W)=H_{G} \exp (W)$ for this involutive semigroup.

Definition A.1. Let $S$ be an involutive semigroup. A multiplier of $S$ is a pair $(\lambda, \rho)$ of maps $\lambda, \rho: S \rightarrow S$ satisfying

$$
a \lambda(b)=\rho(a) b, \quad \lambda(a b)=\lambda(a) b, \quad \text { and } \quad \rho(a b)=a \rho(b) \quad \text { for } \quad a, b \in S .
$$

We write $M(S)$ for the set of all multipliers of $S$ and turn it into an involutive semigroup by

$$
(\lambda, \rho)\left(\lambda^{\prime}, \rho^{\prime}\right):=\left(\lambda \circ \lambda^{\prime}, \rho^{\prime} \circ \rho\right) \quad \text { and } \quad(\lambda, \rho)^{*}:=\left(\rho^{*}, \lambda^{*}\right),
$$

where $\lambda^{*}(a):=\lambda\left(a^{*}\right)^{*}$ and $\rho^{*}(a)=\rho\left(a^{*}\right)^{*}$. We write

$$
\mathrm{U}(M(S)):=\left\{(\lambda, \rho) \in M(S):(\lambda, \rho)(\lambda, \rho)^{*}=(\lambda, \rho)^{*}(\lambda, \rho)=\mathbf{1}\right\}
$$

for the unitary group of $M(S)$.

Note that $S \rightarrow M(S), s \mapsto\left(\lambda_{s}, \rho_{s}\right)$ is a morphism of involutive semigroups and that $M(S)$ acts on $S$ from the left by $(\lambda, \rho) . s:=\lambda(s)$ and from the right by $s .(\lambda, \rho):=\rho(s)$.

The group $H_{G}$ is in general not contained in $S_{H_{G}}(W)$, but it acts on it by the unitary multipliers $\left(\lambda_{h}, \rho_{h}\right), h \in H_{G}$. 
Proposition A.2. Let $q: \widetilde{S} \rightarrow S$ be the universal covering of the Banach manifold $S=S_{H_{G}}(W)$. Then $\widetilde{S}$ carries the structure of an analytic Banach $*$-semigroup such that the covering map $q: \widetilde{S} \rightarrow S$ is a homomorphism of Banach *-semigroups.

Moreover, the simply connected covering group $\widetilde{H}_{G}$ of $H_{G}$ acts on $\widetilde{S}$ by unitary multipliers and we thus obtain an analytic diffeomorphism

$$
\widetilde{\Phi}: \widetilde{H}_{G} \times W \rightarrow \widetilde{S}, \quad(h, x) \mapsto h \exp x,
$$

where $\exp : W \rightarrow \widetilde{S}$ is a continuous lift of $\exp : W \rightarrow S$ such that

$$
\exp (x)^{*}=\exp (x) \quad \text { for } \quad x \in W
$$

and

$$
\exp (s x) \exp (t x)=\exp ((t+s) x) \quad \text { for } \quad x \in W, t, s>0 .
$$

Proof. Since the polar map $\Phi: H_{G} \times W \rightarrow S$ is an analytic diffeomorphism, there exists an analytic diffeomorphism $\widetilde{\Phi}: \widetilde{H}_{G} \times W \rightarrow \widetilde{S}$ with $q \circ \widetilde{\Phi}=\Phi \circ q$. We then define $\widetilde{\exp }: W \rightarrow \widetilde{S}, x \mapsto \widetilde{\Phi}(e, x)$.

Pick $x_{0} \in W$ and let $\widetilde{m}: \widetilde{S} \times \widetilde{S} \rightarrow \widetilde{S},(s, t) \mapsto s t$ be the unique continuous lift of the multiplication map $m: S \times S \rightarrow S$ with

$$
\widetilde{\exp }\left(x_{0}\right) \widetilde{\exp }\left(x_{0}\right)=\widetilde{\exp }\left(2 x_{0}\right) \text {. }
$$

Then the uniqueness of lifts implies that the restriction of $\widetilde{m}$ to $\widetilde{\exp }\left(\mathbb{R}_{>0} x_{0}\right)$ satisfies

$$
\widetilde{\exp }\left(t x_{0}\right) \widetilde{\exp }\left(s x_{0}\right)=\widetilde{\exp }\left((t+s) x_{0}\right)
$$

and we obtain in particular

$$
\left(\widetilde{\exp }\left(x_{0}\right) \widetilde{\exp }\left(x_{0}\right)\right) \widetilde{\exp }\left(x_{0}\right)=\widetilde{\exp }\left(x_{0}\right)\left(\widetilde{\exp }\left(x_{0}\right) \widetilde{\exp }\left(x_{0}\right)\right) \text {. }
$$

Therefore the uniqueness of lifts implies that $\widetilde{m}$ is associative, hence defines on $\widetilde{S}$ an analytic semigroup structure.

We also lift the involution on $S$ to the unique involutive diffeomorphism $*$ on $\widetilde{S}$ with $\widetilde{\exp }\left(x_{0}\right)^{*}=\widetilde{\exp }\left(x_{0}\right)$, and since $(s t)^{*}=t^{*} s^{*}$ now holds for $s=t=\widetilde{\exp }\left(x_{0}\right)$, the uniqueness of lifts implies that $(\widetilde{S}, *)$ is an involutive semigroup.

The multiplication on $S$ can be expressed by analytic maps $m_{H_{G}}: W \times W \rightarrow H_{G}$ and $m_{W}: W \times W \rightarrow W$ as

$$
\Phi(h, x) \Phi\left(h^{\prime}, x^{\prime}\right)=\Phi\left(h h^{\prime} m_{H_{G}}\left(\operatorname{Ad}\left(h^{\prime}\right)^{-1} x, x^{\prime}\right), m_{W}\left(\operatorname{Ad}\left(h^{\prime}\right)^{-1} x, x^{\prime}\right)\right) .
$$

From the continuity of the multiplication $S \times\left(S \cup H_{G}\right) \rightarrow S$ in $G$, it follows that both maps $m_{W}$ and $m_{H}$ extends continuously to the set

$$
W_{2}:=(W \times(W \cup\{0\})) \cup((W \cup\{0\}) \times W) .
$$

If $\widetilde{m}_{H_{G}}: W_{2} \rightarrow \widetilde{H}_{G}$ is the unique lift of $m_{H_{G}}$ satisfying $\widetilde{m}_{H_{G}}\left(x_{0}, x_{0}\right)=e$, then we obtain the formula

$$
\widetilde{\Phi}(h, x) \widetilde{\Phi}\left(h^{\prime}, x^{\prime}\right)=\widetilde{\Phi}\left(h h^{\prime} \widetilde{m}_{H_{G}}\left(\operatorname{Ad}\left(h^{\prime}\right)^{-1} x, x^{\prime}\right), m_{W}\left(\operatorname{Ad}\left(h^{\prime}\right)^{-1} x, x^{\prime}\right)\right) .
$$

For each $x \in W$ and $t, s>0$ we further have $\tilde{m}_{H_{G}}(s x, t x)=e$ because $m_{H_{G}}(s x, t x)=e$ and the subset $\{(s x, t x) \in W \times W: s, t>0, x \in W\}$ is connected. This implies (28).

The left and right multiplier actions of $H_{G}$ on $S$ lift to unique left and right actions of $\widetilde{H}_{G}$ on $\widetilde{S}$, satisfying

$$
h \widetilde{\Phi}\left(h^{\prime}, x\right)=\widetilde{\Phi}\left(h h^{\prime}, x\right)^{\prime} \quad \text { and } \quad \widetilde{\Phi}\left(h^{\prime}, x\right) h=\widetilde{\Phi}\left(h^{\prime} h, \operatorname{Ad}(h)^{-1} x\right) .
$$

From (31) we further derive

$$
(h \widetilde{\exp } x)\left(h^{\prime} \widetilde{\exp } x^{\prime}\right)=h h^{\prime} \widetilde{\exp }\left(\operatorname{Ad}\left(h^{\prime}\right)^{-1} x\right) \widetilde{\exp }\left(x^{\prime}\right) .
$$


This implies in particular that the left action of $\widetilde{H}_{G}$ on $\widetilde{S}$ commutes with the right multiplications. We also obtain from the uniqueness of lifts that

$$
(h \widetilde{\exp } x)^{*}=h^{-1} \widetilde{\exp }(\operatorname{Ad}(h) x)=\widetilde{\exp }(x) h^{-1} \quad \text { for } \quad x \in W, h \in \widetilde{H}_{G},
$$

so that left multiplications in $\widetilde{S}$ commute with the right action of $\widetilde{H}_{G}$. To see that $\widetilde{H}_{G}$ acts on $\widetilde{S}$ by unitary multipliers, it remains to observe that

$$
\left(h^{\prime} \widetilde{\exp }\left(x^{\prime}\right) h\right)\left(h^{\prime \prime} \widetilde{\exp }\left(x^{\prime \prime}\right)\right)=\left(h^{\prime} \widetilde{\exp }\left(x^{\prime}\right)\right)\left(h h^{\prime \prime} \widetilde{\exp }\left(x^{\prime \prime}\right)\right)
$$

for $h, h^{\prime}, h^{\prime \prime} \in \widetilde{H}_{G}, x, x^{\prime} \in W$, which also follows from (32).

Let $\operatorname{Ad}_{\mathfrak{q}} \widetilde{H}_{G}:=\operatorname{Ad}_{\mathfrak{q}} \circ q_{H_{G}}$ be the action of $\widetilde{H}_{G}$ on $\mathfrak{q}$, obtained from the action $\operatorname{Ad}_{\mathfrak{q}}$ of $H_{G}$ on $\mathfrak{q}$ and the covering map $q_{H_{G}}: \widetilde{H}_{G} \rightarrow H_{G}$.

Proposition A.3. For a discrete central subgroup $\Gamma \subseteq \widetilde{H}_{G}$ acting trivially on $\mathfrak{q}$, the cosets in $\widetilde{S}$ satisfy

$$
(s \Gamma)(t \Gamma)=s t \Gamma \quad \text { and } \quad(s \Gamma)^{*}=s^{*} \Gamma \quad \text { for } \quad s, t \in \widetilde{S},
$$

so that the quotient semigroup $\widetilde{S} / \Gamma$ inherits the structure of an analytic involutive Banach semigroup for which the quotient map $q_{\Gamma}: \widetilde{S} \rightarrow \widetilde{S} / \Gamma$ is a morphism of involutive semigroups. Moreover, the polar map $\widetilde{\Phi}: \widetilde{H}_{G} \times W \rightarrow \widetilde{S}$ factors through a diffeomorphism $\Phi_{\Gamma}: \widetilde{H}_{G} / \Gamma \times W \rightarrow \widetilde{S} / \Gamma$ and the group $\widetilde{H}_{G} / \Gamma$ acts faithfully on $\widetilde{S} / \Gamma$ by unitary multipliers.

Proof. Since the left action of $\Gamma$ on $\widetilde{S}$ coincides with the right action (see (31)), the relations (33) easily follow. The remaining assertions are now obvious.

Definition A.4. (a) The semigroups obtained by the preceding proposition will be called Banach-Olshanski semigroups. We write

$$
S_{H}(W):=\widetilde{S}_{H_{G}}(W) / \Gamma \quad \text { for } \quad H=\widetilde{H}_{G} / \Gamma
$$

and $\exp x:=\widetilde{\exp }(x) \cdot \Gamma$ for the exponential function $\exp : W \rightarrow S_{H}(W)$.

(b) For the special case where $\mathfrak{g}=\mathfrak{h}_{\mathbb{C}}$ and $\theta(x+i y)=x-i y$, the group $G$ is complex, so that $\Gamma_{H_{G}}(W)$ is a complex manifold on which the multiplication is holomorphic and the involution is antiholomorphic. These properties are inherited by all other Olshanski semigroups $S_{H}(W)$, where $H \cong \widetilde{H}_{G} / \Gamma$ is a connected Lie group with Lie algebra $\mathfrak{h}$. Therefore we call them complex Olshanski semigroups.

Remark A.5. The basic properties of Olshanski semigroups $S_{H}(W)$ are:

(O1): The polar map $H \times W \rightarrow S_{H}(W),(h, x) \mapsto h \exp x$ is an analytic diffeomorphism.

(O2): $H$ acts on $S_{H}(W)$ smoothly by unitary multipliers.

(O3): For $x \in W$, we have

$$
\exp (s x) \exp (t x)=\exp ((t+s) x) \quad \text { for } \quad t, s>0 .
$$

(O4): For $h \in H$ and $s \in W$, we have $(h \exp x)^{*}=(\exp x) h$.

From now on $H$ always denotes a connected Lie group with Lie algebra $\mathfrak{h}$ and $S=S_{H}(W)$ is a corresponding Olshanski semigroup. We do not assume that $H$ is contained in $G$.

Lemma A.6. For $s \in S_{H}(W)$ and $x \in W$ we have

$$
\lim _{t \rightarrow 0_{+}} \exp (t x) s=\lim _{t \rightarrow 0_{+}} s \exp (t x)=s .
$$


Proof. It suffices to verify this relation in the simply connected covering semigroup $\widetilde{S}$, where we have for $s=h^{\prime} \exp \left(x^{\prime}\right)$ :

$$
\exp (t x) s=\widetilde{\Phi}(e, t x) \widetilde{\Phi}\left(h^{\prime}, x^{\prime}\right)=\widetilde{\Phi}\left(h^{\prime} \widetilde{m}_{H}\left(\operatorname{Ad}\left(h^{\prime}\right)^{-1} t x, x^{\prime}\right), m_{W}\left(\operatorname{Ad}\left(h^{\prime}\right)^{-1} t x, x^{\prime}\right)\right) .
$$

Since the functions $\widetilde{m}_{H}$ and $m_{W}$ extend continuously to the domain $W_{2}$, formula (30) yields $\exp (t x) s \rightarrow s$. The other relation is obtained by applying the involution $*$.

Remark A.7. Let $\kappa^{r} \in \Omega^{1}(G, \mathfrak{g})$ denote the right Maurer-Cartan form, defined by $\kappa_{g}^{r}(x . g):=x$ for $x \in \mathfrak{g}=T_{e}(G)$, where $T G \times G \rightarrow T G,(v, g) \mapsto v \cdot g$ denotes the canonical right action of $G$ on $T G$. Similarly we define the left Maurer-Cartan form by $\kappa_{g}^{l}(g \cdot x):=x$.

For the subsemigroup $S=S_{H_{G}}(W) \subseteq G$, the restriction $\kappa_{S}^{r}:=\left.\kappa^{r}\right|_{S}$ defines a trivialization of the tangent bundle of $S$ by

$$
T S \rightarrow S \times \mathfrak{g}, \quad v_{s} \mapsto\left(s, \kappa_{S}^{r}\left(v_{s}\right)\right) \quad \text { for } \quad v_{s} \in T_{s}(S) .
$$

If $q_{S}: \widetilde{S} \rightarrow S$ is the universal covering, the form $\kappa_{\widetilde{S}}^{r}:=q_{S}^{*} \kappa_{S}^{r}$ likewise trivializes $T(\widetilde{S})$. For every discrete central subgroup $\Gamma \subseteq \widetilde{H}_{G}$ acting trivially on $\mathfrak{q}$ and $S=S_{H_{G}}(W)$, the form $\kappa_{\widetilde{S}}^{r}$ on $\widetilde{S}$ is $\Gamma$-invariant, hence is the pullback of a form $\kappa_{\widetilde{S} / \Gamma}^{r}$ trivializing $T(\widetilde{S} / \Gamma)$.

Definition A.8. The preceding discussion shows that on every Olshanski semigroup $S_{H}(W)$, we have a natural form $\kappa_{S}^{r} \in \Omega^{1}(S, \mathfrak{g})$ trivializing the tangent bundle and we similarly obtain a left invariant form $\kappa_{S}^{l}$.

Accordingly, we have natural left invariant vector fields $V_{x}, x \in \mathfrak{g}$, on $S$, defined by $\kappa_{S}^{l}\left(V_{x}\right)=x$. and right invariant vector fields $W_{x}, x \in \mathfrak{g}$, defined by $\kappa_{S}^{r}\left(W_{x}\right)=x$.

Remark A.9. For $x \in \mathfrak{h} \cup W$ and $s \in S$ we have

$$
V_{x}(s)=\left.\frac{d}{d t}\right|_{t=0} s \exp (t x) \quad \text { and } \quad W_{x}(s)=\left.\frac{d}{d t}\right|_{t=0} \exp (t x) s .
$$

Both relations are obvious for the semigroup $S_{H_{G}}(W)$, and they are inherited by the simply connected covering and hence also by its quotients.

For $x \in \mathfrak{g}$ and $s \in S$, we write $t \mapsto s \exp (t x)$ for the integral curve of $V_{x}$ through $s$ and likewise $t \mapsto \exp (t x) s$ for the local integral curve of $W_{x}$. This is redundant for $S=S_{H_{G}}(W) \subseteq G$, and for a general $S=S_{H}(W)$, the preceding observation shows that it is also consistent for $x \in W \cup \mathfrak{h}$ with the action of the corresponding one-parameter (semi)groups.

Remark A.10. (a) From the right invariance of $\kappa_{G}^{r}$ on $G$, we obtain

$$
\rho_{s}^{*} \kappa_{S}^{r}=\kappa_{S}^{r} \quad \text { for } \quad s \in S=S_{H}(W)
$$

by verifying that this property is preserved by the passage to covering semigroups and to quotients by discrete central subgroups. We likewise get

$$
\lambda_{s}^{*} \kappa_{S}^{l}=\kappa_{S}^{l} \quad \text { for } \quad s \in S .
$$

(b) As in (a), it follows that, for $h \in H$, the right multiplication $\rho_{h}: S \rightarrow S$ also leaves $\kappa_{S}^{r}$ invariant. For the left multiplication $\lambda_{h}(s)=h . s$, the relation $\lambda_{g}^{*} \kappa_{G}^{r}=$ $\operatorname{Ad}(g) \circ \kappa_{G}^{r}$ for the Maurer-Cartan form of a Lie group $G$ implies that

$$
\lambda_{h}^{*} \kappa_{S}^{r}=\operatorname{Ad}(h) \circ \kappa_{S}^{r} \quad \text { for } \quad h \in H .
$$

This is also verified by the passage through the universal covering semigroup. 
Definition A.11. For a smooth map $f: M \rightarrow S=S_{H}(W)$, where $M$ is a smooth manifold, we define the (right) logarithmic derivative as the $\mathfrak{g}$-valued 1-form

$$
\delta(f):=f^{*} \kappa_{S}^{r} \in \Omega^{1}(M, \mathfrak{g})
$$

If $I \subseteq \mathbb{R}$ is an interval and $\alpha: I \rightarrow S$ a differentiable path, then the identification of 1 -forms on $I$ with $\mathfrak{g}$-valued functions leads to

$$
\delta(\alpha)_{t}:=\kappa_{\alpha(t)}^{r}\left(\alpha^{\prime}(t)\right) \in \mathfrak{g} .
$$

We likewise define logarithmic derivatives for maps with values in Lie groups.

For the exponential map exp: $\mathfrak{g} \rightarrow G$ we then have

$$
\delta(\exp )_{x}(y)=\int_{0}^{1} e^{s \operatorname{ad} x} y d s,
$$

([Ne06, Prop. II.5.7]) and therefore:

Proposition A.12. Any $C^{1}$-path $\alpha: I \rightarrow W$ satisfies

$$
\delta(\exp \alpha)_{t}=\int_{0}^{1} e^{s \operatorname{ad} \alpha(t)} \alpha^{\prime}(t) d s .
$$

From the differential of the multiplications, we obtain left and right actions

$$
S \times T S \rightarrow T S, \quad(s, v) \mapsto s . v, \quad T S \times S \rightarrow T S, \quad(v, s) \mapsto v . s
$$

and likewise

$$
H \times T S \rightarrow T S, \quad(h, v) \mapsto h . v, \quad T S \times H \rightarrow T S, \quad(v, h) \mapsto v . h
$$

as well as

$$
T H \times S \rightarrow T S, \quad(v, s) \mapsto v . s, \quad S \times T H \rightarrow T S, \quad(s, v) \mapsto s . v .
$$

We then also have

$$
\left(\kappa_{S}^{r}\right)(v . s)=v \quad \text { for } \quad v \in \mathfrak{h}=T_{e}(H), s \in S
$$

because this is true for the subsemigroup $\Gamma_{H_{G}}(W)$ of $G$.

Proposition A.13. Let $\alpha: I \rightarrow H$ and $\beta: I \rightarrow W$ be two $C^{1}$-paths. For the path $\gamma(t):=\alpha(t) \exp (\beta(t))$ in $S$ we then have

$$
\delta(\gamma)_{t}=\delta(\alpha)_{t}+\operatorname{Ad}(\alpha(t)) \delta(\exp \circ \beta)_{t} .
$$

Proof. First we note that

$$
\gamma^{\prime}(t)=\alpha^{\prime}(t) \cdot \exp \beta(t)+\alpha(t) \cdot(\exp \circ \beta)^{\prime}(t)
$$

To evaluate $\delta(\gamma)_{t}=\left(\kappa_{S}^{r}\right)_{\gamma(t)}\left(\gamma^{\prime}(t)\right)$, we first write $\alpha^{\prime}(t)=\delta(\alpha)_{t} . \alpha(t)$ in $T H$. For $v \in$ $T H$, the relation $\left(h^{\prime} h\right) . s=h^{\prime}$. (h.s) for $s \in S, h, h^{\prime} \in H$ leads to $(v . h) . s=v .(h . s)$, so that (35) implies that $\kappa_{S}^{r}\left(\alpha^{\prime}(t) \cdot \exp \beta(t)\right)=\delta(\alpha)_{t}$. Finally, the relation (34) leads to

$$
\kappa_{S}^{r}\left(\alpha(t) \cdot(\exp \circ \beta)^{\prime}(t)\right)=\operatorname{Ad}(\alpha(t)) \delta(\exp \circ \beta)_{t}
$$




\section{APPENDiX B. FAMILIES OF ONE-PARAMETER SEMIGROUPS}

In this section we let $\mathfrak{g}=(\mathfrak{g},[\cdot, \cdot])$ be a locally convex Lie algebra. This means that $\mathfrak{g}$ is a locally convex space and the Lie bracket $[\cdot, \cdot]$ is continuous. Let us first recall the following basic notions of the differential calculus over locally convex spaces.

Definition B.1. (a) Let $E, F$ be two locally convex spaces, $U$ open and $f: U \subseteq$ $E \rightarrow F$ be a continuous map on the open set $U$ of $E$. Then $f$ is called $C^{1}$ if the directional derivatives $\partial_{v} f(x):=\lim _{h \rightarrow 0} \frac{f(x+h v)-f(x)}{h}$ exist for every $x \in U$ and $v \in E$ and the map

$$
\mathrm{d} f: U \times E \rightarrow F, \quad(x, v) \mapsto \partial_{v} f(x)
$$

is continuous.

(b) A continuous map $f: U \subseteq E \rightarrow V$ is called $C^{k}, k \geq 2$ if it is $C^{1}$ and $\mathrm{d} f$ is $C^{k-1}$. It is called $C^{\infty}$, or smooth, if it is $C^{k}$ for every $k \in \mathbb{N}$.

(c) A locally convex space $E$ is called Mackey complete if for each smooth curve $\xi:[0,1] \rightarrow E$ the weak integral $\int_{0}^{1} \xi(t) d t$ exists, i.e., there exists a (unique) element $I=: \int_{0}^{1} \xi(t) d t \in E$ satisfying

$$
\alpha(I)=\int_{0}^{1} \alpha(\xi(t)) d t \quad \text { for each } \quad \alpha \in E^{\prime} .
$$

This implies in particular that the curve $\eta(s):=\int_{0}^{s} \xi(t) d t$ is smooth and satisfies $\eta^{\prime}=\xi$.

Definition B.2. A locally convex Lie algebra $\mathfrak{g}$ is called ad-integrable if for every $x \in \mathfrak{g}$ the (linear) vector field defined by ad $x$ is complete, that is, if there exists a smooth map $\Phi^{x}: \mathbb{R} \times \mathfrak{g} \rightarrow \mathfrak{g}$ with $\left.\frac{d}{d t}\right|_{t=0} \Phi^{x}(t) y=\operatorname{ad} x(y)$. We will then use the notation $e^{t \operatorname{ad} x}:=\Phi^{x}(t)$.

We consider a linear homomorphism

$$
\alpha: \mathfrak{g} \rightarrow \operatorname{End}(\mathcal{D})
$$

in the space of endomorphism of a dense domain $\mathcal{D}$ of a Banach space $E$. We assume that $\alpha$ is strongly continuous in the sense that for every $v \in \mathcal{D}$ the map $\alpha^{v}: \mathfrak{g} \rightarrow E, x \mapsto \alpha(x) v$ is continuous. Now let $W$ be a convex cone in $\mathfrak{g}$ which is relatively open in its span $\mathfrak{q}:=W-W$ (cf. Definition 5.1), and assume that, for every $x \in W$, the closure $\overline{\alpha(x)}$ of $\alpha(x)$ generates a strongly continuous semigroup $\left(e^{\overline{t \overline{\alpha(x)}}}\right)_{t \geq 0}$ and that the map $s_{\alpha}(x):=\sup _{0 \leq t \leq 1}\left\|e^{t \overline{\alpha(x)}}\right\|$ is locally bounded on $W$.

Remark B.3. If $E=\mathcal{H}$ is a Hilbert and for every $x \in W$ the semigroup $\left(e^{t \overline{\alpha(x)}}\right)_{t>0}$ consists of normal operators, then the fact it generates a commutative $C^{*}$-algebra isomorphic to some $C(X)$ implies that

$$
\left\|e^{t \overline{\alpha(x)}}\right\|=\left\|e^{\overline{\alpha(x)}}\right\|^{t} \quad \text { for } x \in W,
$$

and hence that $s_{\alpha}(x)=\max \left\{1,\left\|e^{\overline{\alpha(x)}}\right\|\right\}$.

We will need the following lemma (see [Mer10, Lemma 9]):

Lemma B.4. Consider two operators $A$ and $B$ defined on a common dense domain $\mathcal{D}$ of the Banach space $E$ and whose closures generates strongly continuous 
semigroups $\left(e^{t \bar{A}}\right)_{t>0}$ and $\left(e^{t \bar{B}}\right)_{t>0}$ respectively. Assume further that $e^{s A} \mathcal{D} \subseteq \mathcal{D}$ for all $s \geq 0$. If, for some $v \in \mathcal{D}$ the map $s \mapsto B e^{s A} v$ is continuous on $[0, \infty)$, then

$$
e^{t \bar{B}} v-e^{t \bar{A}} v=\int_{0}^{t} e^{s \bar{B}}(B-A) e^{(t-s) \bar{A}} v d s \quad \text { for } \quad t \geq 0 .
$$

We then have the following proposition generalizing [Mer10, Prop. 10]:

Proposition B.5. Let $\mathfrak{g}$ be an ad-integrable Mackey complete locally convex Lie algebra. If, for every $x \in W$ and every $y \in \mathfrak{g}$,

$$
e^{\overline{\alpha(x)}} \mathcal{D} \subseteq \mathcal{D} \quad \text { and } \quad \alpha(y) e^{\overline{\alpha(x)}}=e^{\overline{\alpha(x)}} \alpha\left(e^{-\operatorname{ad} x} y\right),
$$

then the map

$$
\widehat{\rho}: W \times E \rightarrow E, \quad(x, v) \mapsto \rho^{v}(x):=e^{\overline{\alpha(x)}} v
$$

is continuous, and for every $v \in \mathcal{D}$, $\rho^{v}$ is $C^{1}$ with

$$
T_{x}\left(\rho^{v}\right)(y)=\alpha\left(\int_{0}^{1} e^{s \operatorname{ad} x} y d s\right) e^{\overline{\alpha(x)}} v=e^{\overline{\alpha(x)}} \alpha\left(\int_{0}^{1} e^{-s \operatorname{ad} x} y d s\right) v .
$$

Proof. Let $v \in \mathcal{D}$ and $x, y \in \mathfrak{g}$. From the relation

$$
\alpha(y) e^{s \overline{\alpha(x)}} v=e^{s \overline{\alpha(x)}} \alpha\left(e^{-s \operatorname{ad} x} y\right) v,
$$

the continuity of the map $\left[0, \infty\left[\times E \rightarrow E,(s, v) \mapsto e^{s \overline{\alpha(x)}} v\right.\right.$ and the strong continuity of $\alpha$, we derive that $s \mapsto \alpha(y) e^{s \overline{\alpha(x)}} v$ is continuous on [0, [. We can therefore apply Lemma B.4 to obtain

$$
\begin{gathered}
e^{\overline{\alpha(y)}} v-e^{\overline{\alpha(x)}} v=\int_{0}^{1} e^{u \overline{\alpha(y)}} \alpha(y-x) e^{(1-u) \overline{\alpha(x)}} v d u \\
=\int_{0}^{1} e^{u \overline{\alpha(y)}} e^{(1-u) \overline{\alpha(x)}} \alpha\left(e^{(u-1) \operatorname{ad} x}(y-x)\right) v d u .
\end{gathered}
$$

Let $\varepsilon>0$ let $\mathcal{V}$ be a 0 -neighbourhood in $\mathfrak{g}$ such that for every $y \in x+\mathcal{V}, s_{\alpha}(y)<M$. Consider now the continuous map

$$
F:[0,1] \times \mathfrak{g} \rightarrow E,(u, z) \mapsto \alpha^{v}\left(e^{(u-1) \text { ad } x} z\right) .
$$

Since $F([0,1] \times\{0\})=\{0\}$, the compactness of $[0,1]$ implies the existence of a 0 neighborhood $\mathcal{V}^{\prime} \subseteq \mathfrak{g}$ such that $F\left([0,1] \times \mathcal{V}^{\prime}\right)$ is contained in the open ball $B\left(0, \frac{M}{\varepsilon}\right)$ of radius $\frac{M}{\varepsilon}$ around 0 in $E$. Thus, for every $y \in x+\mathcal{V} \cap \mathcal{V}^{\prime},\left|e^{\overline{\alpha(y)}} v-e^{\overline{\alpha(x)}} v\right| \leq \varepsilon$, and this proves that $\rho^{v}$ is continuous. The local boundedness of $s_{\alpha}: W \rightarrow \mathbb{R}$ further implies that $\rho^{v}$ is continuous for every $v \in E$, and hence the map

$$
\widehat{\rho}: W \times E \rightarrow E,(x, v) \mapsto e^{\overline{\alpha(x)}} v
$$

is continuous.

Let $y \in \mathfrak{q}$ and let $\tau>0$ such that $x+h y \in W$ for $|h|<\tau$. We derive from (38) the formula

$$
\frac{e^{\overline{\alpha(x+h y)}} v-e^{\overline{\alpha(x)}} v}{h}=\int_{0}^{1} e^{s \overline{\alpha(x+h y)}} \alpha(y) e^{(1-s) \overline{\alpha(x)}} v d s .
$$

Let us fix $0<\varepsilon \leq 1$. Then the continuity of the map

$$
(s, h) \mapsto e^{s \overline{\alpha(x+h y)}} \alpha(y) e^{(1-s) \overline{\alpha(x)}} v
$$

on $[\varepsilon, 1] \times[-\tau, \tau]$ implies that we can pass to the limit under the integral sign to derive

$$
\lim _{h \rightarrow 0} \int_{\varepsilon}^{1} e^{s \overline{\alpha(x+h y)}} \alpha(y) e^{(1-s) \overline{\alpha(x)}} v d s=\int_{\varepsilon}^{1} e^{s \overline{\alpha(x)}} \alpha(y) e^{(1-s) \overline{\alpha(x)}} v d s .
$$


The same type of argument as the one used for the continuity of $\rho^{v}$ shows that the integrand of

$$
\int_{0}^{\varepsilon} e^{\overline{s(x+h y)}} \alpha(y) e^{(1-s) \overline{\alpha(x)}} v d s
$$

is bounded unformly with respect to $(s, h)$, and hence the integral is uniformly small (with respect to $h$ ) when $\varepsilon$ is sufficently close to 0 . Therefore

$$
\begin{aligned}
\left(\partial_{y} \rho^{v}\right)(x) & =\int_{0}^{1} e^{s \overline{\alpha(x)}} \alpha(y) e^{(1-s) \overline{\alpha(x)}} v d s=\int_{0}^{1} \alpha\left(e^{s \text { ad } x} y\right) e^{\overline{\alpha(x)}} v d s \\
& =\alpha\left(\int_{0}^{1} e^{s \text { ad } x} y d s\right) e^{\overline{\alpha(x)}} v
\end{aligned}
$$

where the last equality follows from the uniqueness of the weak integral. Similarly we obtain

$$
\partial_{y} \rho^{v}(x)=e^{\overline{\alpha(x)}} \alpha\left(\int_{0}^{1} e^{-s \operatorname{ad} x} y d s\right) v,
$$

and now the continuity of $\widehat{\rho}$ implies that $T \rho^{v}: W \times \mathfrak{q} \rightarrow E$ is continuous, i.e, that $\rho^{v}$ is a $C^{1}$-map.

\section{REFERENCES}

[EN00] Engel, K.-J., and R. Nagel, "One-parameter Semigroups for Linear Evolution Equations," Graduate Texts in Mathematics 194, Springer-Verlag, New York, 2000.

[Frö80] Fröhlich, J., Unbounded, symmetric semigroups on a separable Hilbert space are essentially selfadjoint, Adv. in Appl. Math. 1 (1980), no. 3, 237-256.

[FOS83] Fröhlich, J., Osterwalder, K., and E. Seiler, On virtual representations of symmetric spaces and their analytic continuation, Annals Math. 118 (1983), 461-489

[Ga60] Gårding, L., Vecteurs analytiques dans les représentations des groupes de Lie, Bull. Soc. Math. France 88 (1960), 73-93

[GJ81] Glimm, J., and A. Jaffe, "Quantum Physics-A Functional Integral Point of View," Springer-Verlag, New York, 1981

[Ham82] Hamilton, R., The inverse function theorem of Nash and Moser, Bull. Amer. Math. Soc. 7 (1982), 65-222

[HN93] Hilgert, J., and K.-H. Neeb, "Lie Semigroups and their Applications," Lecture Notes in Mathematics 1552, Springer-Verlag, Berlin, 1993

[JO100] Jorgensen, P. E. T., and G. Ólafsson, Unitary representations and OsterwalderSchrader duality, in "The Mathematical Legacy of Harish-Chandra," R. S. Doran and V. S. Varadarajan, eds., Proc. Symp. in Pure Math. 68, Amer. Math. Soc., 2000

[Kat66] Kato, T., "Perturbation Theory for Linear Operators," Grundlehren der mathematischen Wissenschaften 132, Springer-Verlag, New York, 1966

[Kau83] Kaup, W., Über die Klassifikation der symmetrischen hermiteschen Mannigfaltigkeiten unendlicher Dimension I, II, Math. Annalen 257 (1981), 463-486; 262(1983), 57-75

[Kau97] _ - On real Cartan factors, manuscripta math. 92 (1997), 191-222

[La94] Lawson, J. D., Polar and Ol'shanskiǔ decompositions, J. Reine Angew. Math. 448 (1994), $191-219$

[LM75] Lüscher, M., and G. Mack, Global conformal invariance and quantum field theory, Comm. Math. Phys. 41 (1975), 203-234

[Mer10] Merigon, S., Integrating representations of Banach-Lie algebras, J. Funct. Anal. 260 (2011), 1463-1475

[MN11] Merigon, S., and K.-H. Neeb, Unitary representations of automorphism groups of real Hilbert domains, in preparation

[Ne92] Neeb, K.-H., On the fundamental group of a Lie semigroup, Glasgow Math. J. 34 (1992), 379-394

[Ne00] - "Holomorphy and Convexity in Lie Theory," de Gruyter, Expositions in Mathematics 28, Walter de Gruyter \& Co., Berlin, 2000.

[Ne01] - Compressions of infinite dimensional bounded symmetric domains, Semigroup Forum 63:1 (2001), 71-105

[Ne02] —, A Cartan-Hadamard Theorem for Banach-Finsler manifolds, Geometriae Dedicata 95 (2002), 115-156

[Ne06] -, Towards a Lie theory of locally convex groups, Jap. J. Math. 1:2 (2006), 291-468 
[Ne08] - A complex semigroup approach to group algebras of infinite dimensional Lie groups, Semigroup Forum 77 (2008), 5-35

[Ne10a] - On Analytic Vectors for Representations of Banach-Lie Groups, Ann. Inst. Fourier, to appear; arXiv:math.RT.1002.4792v1 (25 Feb 2010)

[Ne10b] -, On Differentiable Vectors for Representations of Infinite Dimensional Lie Groups, J. Funct. Anal. 259 (2010), 2814-2855; arXiv:math.RT.1002.1602v1 (8 Feb 2010)

[Ne10c] - Semibounded representations of infinite dimensional hermitian groups, in preparation

[NO11] Neeb, K.-H., and G. Ólafsson, Reflection Positivity and Conformal Symmetry, in preparation

[Ol82] Olshanski, G. I., Invariant cones in Lie algebras, Lie semigroups, and the holomorphic discrete series, Funct. Anal. Appl. 15 (1982), 275-285.

[RS80] Reed, M., and B. Simon, "Methods of Modern Mathematical Physics. I, Functional Analysis" 2nd ed., Academic Press Inc., New York, 1980

[Ru87] Rudin, W., "Real and Complex Analysis,"3rd ed., McGraw-Hill Book Co., New York, 1987

Department Mathematik, FAU Erlangen-Nürnberg, Bismarckstrasse 1 1/2, 91054Erlangen, Germany; neeb@Mi.uni-ERlangen.de 